\title{
Firing Rate Homeostasis Can Occur in the Absence of Neuronal Activity-Regulated Transcription
}

\author{
๑Delsey M. Tyssowski, ${ }^{1}$ Katherine C. Letai, ${ }^{1}$ Samuel D. Rendall, ${ }^{\dagger}{ }^{\dagger}$ Chao Tan, ${ }^{2}$ Anastasia Nizhnik, ${ }^{1}$ Pascal S. Kaeser, ${ }^{2}$ \\ and Jesse M. Gray ${ }^{1}$ \\ Departments of ${ }^{1}$ Genetics and ${ }^{2}$ Neurobiology, Harvard Medical School, Boston, Massachusetts 02115
}

Despite dynamic inputs, neuronal circuits maintain relatively stable firing rates over long periods. This maintenance of firing rate, or firing rate homeostasis, is likely mediated by homeostatic mechanisms such as synaptic scaling and regulation of intrinsic excitability. Because some of these homeostatic mechanisms depend on transcription of activity-regulated genes, including Arc and Homerla, we hypothesized that activity-regulated transcription would be required for firing rate homeostasis. Surprisingly, however, we found that cultured mouse cortical neurons from both sexes grown on multi-electrode arrays homeostatically adapt their firing rates to persistent pharmacological stimulation even when activity-regulated transcription is disrupted. Specifically, we observed firing rate homeostasis in Arc knock-out neurons, as well as knock-out neurons lacking the activity-regulated transcription factors AP1 and SRF. Firing rate homeostasis also occurred normally during acute pharmacological blockade of transcription. Thus, firing rate homeostasis in response to increased neuronal activity can occur in the absence of neuronal-activity-regulated transcription.

Key words: activity-dependent transcription; activity-regulated genes; firing rate homeostasis; homeostatic plasticity

\section{Significance Statement}

Neuronal circuits maintain relatively stable firing rates even in the face of dynamic circuit inputs. Understanding the molecular mechanisms that enable this firing rate homeostasis could potentially provide insight into neuronal diseases that present with an imbalance of excitation and inhibition. It has long been proposed that activity-regulated transcription could underlie firing rate homeostasis because activity-regulated genes turn on when neurons are above their target firing rates and include many genes that could regulate firing rate. Surprisingly, despite this prediction, we found that cortical neurons can undergo firing rate homeostasis in the absence of activity-regulated transcription, indicating that firing rate homeostasis can be controlled by non-transcriptional mechanisms.

\section{Introduction}

Neuronal circuits maintain relatively stable firing rates even in the face of changes in circuit inputs. For example, upon eliminating visual input to the mouse visual cortex via monocular deprivation, the firing rates of visual cortex neurons initially decrease, but over a period of 3-4 d, they return to the pre-deprived levels (Hengen et al., 2013, 2016), thus undergoing homeostasis. Firing

Received May 14, 2019; revised Sept. 27, 2019; accepted 0ct. 18, 2019.

Author contributions: K.M.T., K.C.L., S.D.R., C.T., P.S.K., and J.M.G. designed research; K.M.T., K.C.L., S.D.R., C.T., and A.N. performed research; S.D.R. contributed unpublished reagents/analytic tools; K.M.T., K.C.L., S.D.R., and C.T. analyzed data; K.M.T. and K.C.L. wrote the paper.

This work was supported by Grants from the NIH (MH116223 to J.M.G., MH113349 to P.S.K.), the HarvardArmenise Foundation (to J.M.G.), by the Kaneb family (to J.M.G.), and by the National Science Foundation Graduate Research Fellowship Program (DGE1144152 and DEG1745303 to K.M.T.). We thank Yasmin Escobedo Lozoya and members of the Gray laboratory for feedback throughout the project.

The authors declare no competing financial interests.

'Deceased, Dec 2018.

Correspondence should be addressed to Jesse M. Gray at gray@genetics.med.harvard.edu.

https://doi.org/10.1523/JNEUROSCI.1108-19.2019

Copyright $\odot 2019$ the authors rate homeostasis has also been observed in cultured neurons in response to chronic stimulation or chronic blockade of neuronal activity (Turrigiano et al., 1998; Burrone et al., 2002; Bateup et al., 2013; Pozzi et al., 2013; Slomowitz et al., 2015). Understanding the molecular mechanisms that enable firing rate homeostasis may provide insight into human diseases that present with an imbalance of excitation and inhibition in the brain, such as epilepsy or autism (Turrigiano, 2011). However, the molecular mechanisms underlying firing rate homeostasis are largely unknown.

We hypothesized that activity-regulated transcription would be required for firing rate homeostasis because it is required for several forms of homeostatic plasticity that could regulate firing rate. First, synaptic scaling, a multiplicative homeostatic change in the number of AMPA receptors at synapses (Turrigiano et al., 1998), is impaired in one direction (scaling down) by the loss of the activity-regulated genes (ARGs) Homer1a and Plk2, and it is impaired in the other direction (scaling up) by both acute blockade of transcription and by loss of the ARGs Arc, Nptx1, Plk2, and 
Homer1a (Chowdhury et al., 2006; Shepherd et al., 2006; Ibata et al., 2008; Seeburg et al., 2008; Hu et al., 2010; Diering et al., 2017; Schaukowitch et al., 2017). Activity-regulated transcription also homeostatically regulates excitatory/inhibitory (E/I) balance, or the relative strengths and numbers of excitatory and inhibitory synapses. Acute blockade of transcription following stimulation impairs homeostatic decreases in excitatory synapse number (Goold and Nicoll, 2010), and ARGs, including Nptx2, Igf1, Bdnf, and Npas4, homeostatically regulate inhibitory synapse number (Chang et al., 2010; Bloodgood et al., 2013; Spiegel et al., 2014; Hartzell et al., 2018; Gray and Spiegel, 2019). Finally, transcription mediates homeostatic changes in intrinsic excitability, that is, a neuron's likelihood of firing an action potential (Turrigiano, 2011). In the Drosophila neuromuscular junction, the transcription factor Kruppel is required for homeostatic alterations in intrinsic excitability, likely because of its regulation of potassium channels (Parrish et al., 2014; Kulik et al., 2019). Although it is unclear whether Kruppel regulates activity-dependent or basal transcription, the mammalian ARG program has an enrichment for potassium channels (Cho et al., 2016), suggesting that activity-dependent potassium channel transcription may homeostatically change intrinsic excitability. Importantly, each of these transcription-dependent forms of homeostatic plasticitysynaptic scaling, changing E/I balance, and changing intrinsic excitability — can alter firing rates and are thus leading candidate mechanisms underlying firing rate homeostasis (Turrigiano, 2012). Consistent with this idea, synaptic scaling and homeostatic changes in intrinsic excitability both occur in neurons undergoing firing rate homeostasis (Turrigiano et al., 1998; Burrone et al., 2002; Bateup et al., 2013; Hengen et al., 2013; Slomowitz et al., 2015). Thus, we reasoned that transcription might regulate firing rate homeostasis through one or more of these forms of homeostatic plasticity thought to underlie firing rate homeostasis.

In addition, ARG transcription and firing rate homeostasis occur over a period of several hours following chronic stimulation or activity blockade (Bateup et al., 2013; Hengen et al., 2013; Slomowitz et al., 2015; Schaukowitch et al., 2017; Tyssowski et al., 2018; Yap and Greenberg, 2018). Indeed, a model that takes into account the kinetics of ARG induction and the mechanisms mediating their induction suggests that activity-regulated transcription of ion channels could regulate firing rate homeostasis (O'Leary et al., 2014). Therefore, we considered activity-regulated transcription to be a strong candidate regulator of firing rate homeostasis based on both the composition of the ARG program and the kinetics of ARG induction. However, in the present study we observe firing rate homeostasis even when activity-regulated transcription is disrupted, indicating that firing rate homeostasis can occur in the absence of activity-regulated transcription.

\section{Materials and Methods}

Animal care. All animal care and experimental procedures were approved by the Institutional Animal Care and Use Committees at Harvard Medical School. Animals were housed with standard mouse chow and water provided ad libitum.

Mouse (Mus musculus) strains. Wild-type CD-1 litters were acquired from Charles River Laboratories. The Arc KO strain (007662, The Jackson Laboratory) expresses a destabilized form of GFP in the place of Arc under the Arc promoter (Wang et al., 2006). The SRF KO strain (The Jackson Laboratory, 006658) contains loxP sites flanking the promoter and exon 1 sequences of SRF. The AP1 KO strain is a triple-transgenic acquired from the Greenberg Laboratory (Vierbuchen et al., 2017), con- tains loxP sites at three AP1 subunits: Fos (Fleischmann et al., 2003), Fosb (created by Vierbuchen et al., 2017), and Junb (Kenner et al., 2004).

Neuronal culture. Cortical neurons were dissected from pups of mixed sex on postnatal days $0-2$ and dissociated with papain $[(\mathrm{L})(\mathrm{S}) 003126$, Worthington]. Neurons were plated on standard plastic plates coated with poly-ornithine (30 $\mu \mathrm{g} / \mathrm{ml}$; Sigma-Aldrich) in water, or on Lumos MEA plates (Axion Biosystems) or Lumos OptiClear plates (Axion Biosystems) coated with poly-ornithine $(30 \mu \mathrm{g} / \mathrm{ml})$ and $5 \mu \mathrm{g} / \mathrm{ml} \mathrm{laminin}$ (Invitrogen). Neurons were cultured in BrainPhys media (Stem Cell Technologies), supplemented with SM1 (Stem Cell Technologies), penicillin-streptomycin (Invitrogen), and fungizone (Gemini Bio). Cultures were incubated at $37^{\circ} \mathrm{C}$ in $5 \% \mathrm{CO}_{2}$. Experiments were performed on neurons between 15 and 28 DIV. For conditional knock-out (KO) experiments (SRF and AP1), neurons were treated with homemade AAV-CaMKII-GFP or AAV-CaMKII-Cre at 3-4 DIV for $3 \mathrm{~d}$, and experiments were performed at least $14 \mathrm{~d}$ following viral treatment.

Viral production. AAV-CaMKII-Cre and AAV CaMKII-eGFP virus were made by transfecting HEK293T cells with the particles pHelper, RC1, RC2, and CaMKII-Cre or CaMKII-eGFP. Viral supernatant was collected on Day 3 of transfection, by collecting viral supernatant after 3 freeze/thaw cycles at $-80^{\circ} \mathrm{C}$ and $37^{\circ} \mathrm{C}$. Cellular debris was removed by centrifugation at for $1500 \times g$ for $15 \mathrm{~min}$, and filtering through a $0.45 \mu \mathrm{m}$ filter. Virus was stored at $4^{\circ} \mathrm{C}$ for up to 1 month.

$M E A$ recordings. Recordings were made using the Maestro and MiddleMan from Axion Biosystems (version 1.0.0.0), along with Axion's AxIS software v2.4.5. Lumos MEA plates have 48 wells, each containing 16 PEDOT electrodes in a $4 \times 4$ grid. Electrodes are $50 \mu \mathrm{m}$ in diameter and spaced $350 \mu \mathrm{m}$ apart. Neurons were kept at $37^{\circ} \mathrm{C}$ with $5 \% \mathrm{CO}_{2}$ during recordings using the Axion Maestro system.

Spike sorting. Spike sorting was performed similarly to other published in vitro studies (Fong et al., 2015; Slomowitz et al., 2015). Specifically, raw data were filtered in AxIS online using a $200 \mathrm{~Hz}$ Butterworth high-pass filter and a $3000 \mathrm{~Hz}$ Butterworth low-pass filter. Spikes were detected in AxIS online using peak detection with an adaptive threshold of 5.5 standard deviations from noise levels. To avoid detection of overlapping spikes, detection was prevented for $2.16 \mathrm{~ms}$ after each peak.

Spikes were semiautomatically sorted offline in MATLAB using custom-written code clustering waveforms (vectors of voltage over time, detected by AxIS) in principal component (PC) space and clustered by fitting to Gaussian mixture models. The number of clusters was determined manually using a graphical user interface (GUI) that allows the user to see the spikes plotted in PC space as well as the spike traces and mean spike waveforms for each cluster. We rarely observed spikes with an atypical shape, and those that we did observe were at low-frequency and therefore filtered out later in our analysis. Furthermore, upon the addition of tetrodotoxin (TTX; $1 \mu \mathrm{M}$ ) at the end of several experiments, we observed a complete cessation of spiking, indicating that the spikes we identified were a result of neuronal activity. We also very rarely observed single clusters that appeared to consist of two distinct spike waveforms, and we eliminated those from our analysis. Finally, we sometimes observed two clusters that were separated in PC space and by Gaussian mixture modeling that shared the same spike waveform, as determined by visual inspection. In those cases, we considered those spikes to be a single unit. (Most of these cases were mirror image spikes, likely from an axon crossing the electrode and picking up spikes from both sides.)

To ensure that we were only analyzing units that we were able to accurately trace over time, we filtered out units that did not maintain a firing rate of at least $0.001 \mathrm{~Hz}$ for at least $28 \mathrm{~h}$ of the experiment (see MEA data analysis). We also visually confirmed using the GUI that the spike waveforms in each unit did not change dramatically over time, which could indicate that the unit includes two different neurons: one active early in the recording and one active later.

Homeostasis assay. In each experiment, the microelectrode array (MEA) plate was moved to the Maestro at least $2 \mathrm{~h}$ before recording baseline firing rate, to exclude from the recording any activity changes resulting from moving the plate from the incubator to the machine. The baseline recording lasted $6 \mathrm{~h}$, and then picrotoxin (PTX; $2.5 \mu \mathrm{M}$ in DMSO and water; Tocris Bioscience) or DMSO (1:1000 in water) was added to individual wells and activity was recorded for an additional $30 \mathrm{~h}$. DMSO- 
Table 1. Alternative stimulations attempted

\begin{tabular}{|c|c|c|c|}
\hline Drug & Mechanism of action & Concentration & Results \\
\hline Dendrotoxin & Blocks $K+$ channels $\left(K_{v} 1.1, K_{v} 1.2, K_{v} 1.6\right)$ & $10 \mathrm{~nm}, 100 \mathrm{~nm}$ & Increases FR, but adaptation is inconsistent \\
\hline 4-Aminopyridine & Blocks $K+$ channels $\left(K_{v} 1.1, K_{v} 1.2\right)$ & $10-100 \mu \mathrm{M}$ & Increases FR, but adaptation is inconsistent \\
\hline Barium & $\begin{array}{l}\text { Activates calcium-dependent } K+\text { current, and blocks voltage-dependent } \\
K+\text { current and } K+\text { leak currents }\end{array}$ & $0.1 \mathrm{~mm}$ & Increases FR, but does not adapt \\
\hline $\mathrm{KCl}$ & Increases extracellular $\mathrm{K}^{+}$ & $0.1 \mathrm{~mm}$ & Does not consistently increase FR \\
\hline Sodium & Increases extracellular $\mathrm{Na}{ }^{+}$ & $60 \mathrm{~mm}$ & Does not increase FR \\
\hline $0 D 1$ & Activates $\mathrm{Na}+$ channels $\left(\mathrm{Na}_{\mathrm{v}} 1.6, \mathrm{Na}_{\mathrm{v}} 1.7\right)$ & $10-300 \mathrm{~nm}$ & Does not increase FR \\
\hline Bicuculline & $\mathrm{GABA}_{\mathrm{A}}$ antagonist & $2-40 \mu \mathrm{M}$ & Increases FR, but duration and magnitude of the increase is variable \\
\hline Cyclothiazide & AMPA receptor agonist & $50 \mu \mathrm{M}, 100 \mu \mathrm{M}$ & Drug loses potency in $0.5 \mathrm{~d}$ \\
\hline
\end{tabular}

treated neurons received twice the amount of DMSO as PTX-treated neurons. In actinomycin D (ActD) experiments, ActD $(1 \mu \mathrm{g} / \mathrm{ml})$ was added to individual wells for 30 min before PTX or DMSO. All drugs remained in the cultures for the duration of the $30 \mathrm{~h}$ experiment.

One replicate each for $A r c K O, S R F$ conditional knock-out (cKO), and $A P 1 \mathrm{cKO}$ were treated with $1 \mu \mathrm{M}$ PTX instead of $2.5 \mu \mathrm{M}$ PTX. However, the poststimulus fold induction of firing rate in this replicate was comparable to replicates receiving $2.5 \mu \mathrm{M}$ PTX treatment, so we included the replicate in our analysis.

While refining this assay, we tried stimulating activity with other drugs in place of PTX, with a range of success. We chose to focus on PTX because it most consistently caused an increase in firing rate and subsequent homeostasis of firing rate. The other drugs attempted, along with a brief description of their effects on activity, can be found in Table 1 .

MEA data analysis. Spike data were binned into $5 \mathrm{~min}$ bins in MATLAB (MathWorks) and then analyzed further in Python and R. Median firing rate was determined for each experimental condition by taking the median firing rate of all neurons (across multiple wells) at each time bin. Baseline firing rate, post-stimulation firing rate, and final firing rate were determined for each condition by calculating the average median firing rate in the last $3 \mathrm{~h}$ of baseline, $30 \mathrm{~min}$ to $3 \mathrm{~h} 30 \mathrm{~min}$ after adding PTX, and the last $3 \mathrm{~h}$ of the $30 \mathrm{~h}$ PTX exposure (respectively).

We included in the analysis only units whose firing rates were stably $>0.001 \mathrm{~Hz}$ for at least $28 \mathrm{~h}$ of the experiment. PTX-treated units were excluded if their poststimulation firing rate was not greater than baseline firing rate because we cannot assess firing rate homeostasis in neurons that do not increase their firing rate. This filter excluded an average of $44 \%$ of units. We confirmed that performing the same filtering on DMSO-treated cultures did not result in a similar increase in median firing rate in the first $3 \mathrm{~h}$ after adding DMSO, indicating that the observed increase in firing rate is indeed due to PTX treatment and not an artifact of our filtering. Cultures treated with DMSO and filtered for units that increased upon DMSO addition showed a median 1.27-fold change in firing rate, which is significantly less than the median 2.78 -fold change we observed after the same filtering in cultures treated with PTX ( $p=0.01$, two-sided paired $t$ test). The data plotted show unfiltered DMSO units because we are not directly comparing DMSO and PTX treatments. We also confirmed that average proportion of units excluded did not differ between genotypes or drug treatments using a paired rank sum test [Arc $+/-$ vs $-/-: p=1$, SRF control vs KO: $p=1$, AP1 control vs KO: $p=$ 0.8125 , ActD vs control: $p=0.625$, flavopirodol (FLA) vs control: $p=$ $0.25]$. Next, if the poststimulation firing rate of a single PTX-treated well was not at least 1.3-fold of baseline firing rate, that well's response was considered too weak and it was excluded from analysis. We excluded wells in $67.5 \%$ of experiments. We used an average of seven wells per condition and excluded a median of $17 \%$ of wells each experiment. We confirmed that the percentage of wells excluded was not different between genotypes or drug treatments using a paired rank sum test (Arc $+/-$ vs $-/-: p=1$, SRF control vs KO: $p=0.875$, AP1 control vs KO: $p=0.06$, ActD vs control: $p=1$, FLA vs control: $p=1$ ). An entire experiment was excluded if the median firing rate of any $5 \mathrm{~h}$ period in the DMSO control condition was $<0.75$-fold or $>1.3$-fold of baseline firing rate.

To determine the length of time it took for median firing rates to achieve homeostasis, we first calculated a rolling SD of the median firing rate over $6 \mathrm{~h}$ bins. We then determined homeostasis firing rate, which is the firing rate at the time point after PTX addition where the rolling SD was lowest. We said that the median had achieved homeostasis at the time point post-PTX when the median firing rate first reaches at least $102.5 \%$ of the homeostasis firing rate.

Plots of median firing rate over time (Fig. $1 B$ ) are data from 5 min bins smoothed with the lowess function in $\mathrm{R}$ with $\mathrm{f}$ set at $1 / 20$.

Code accessibility. Custom code for both spike sorting and analysis is available on GitHub at https://github.com/kletai/mea_analysis_homeostasis. The MATLAB portions of this analysis were conducted on the $\mathrm{O} 2$ High Performance Compute Cluster, supported by the Research Computing Group, at Harvard Medical School. For more information, see http://rc.hms.harvard.edu.

Whole-cell electrophysiological recordings. Whole-cell recordings in cultured cortical neurons were performed at 15-17 DIV at room temperature as described previously (Held et al., 2016) with small modifications. Neurons were incubated with PTX (2.5 $\mu \mathrm{M}$ in DMSO) or DMSO alone for $15-20 \mathrm{~h}$ before the electrophysiological recordings. The extracellular solution contained the following (in $\mathrm{mM}$ ): $140 \mathrm{NaCl}, 5 \mathrm{KCl}, 2 \mathrm{CaCl}_{2}, 2$ $\mathrm{MgCl}_{2}, 10$ HEPES-NaOH, pH 7.4, 10 glucose ( 210 mOsm). TTX (1 $\mu \mathrm{M})$, PTX (50 $\mu \mathrm{M})$, and D-(-)-2-amino-5-phosphonopentanoic acid (50 $\mu \mathrm{M})$ were added to the extracellular solution to block action potentials, $\mathrm{GABA}_{\mathrm{A}}$ receptors, and NMDA receptors, respectively. Glass pipettes for patch clamping were pulled at 2-5 $\mathrm{M} \Omega$ and filled with intracellular solution containing the following (in $\mathrm{mM}$ ): $120 \mathrm{Cs}$-methanesulfonate, 10 EGTA, $2 \mathrm{MgCl}_{2}, 10$ HEPES-CsOH, pH 7.4, $4 \mathrm{Na}_{2}$-ATP, 1 Na-GTP, 4 QX314-Cl ( $\sim 300 \mathrm{mOsm})$. Data were acquired with an Axon 700B MultiClamp amplifier and digitized with a Digidata 1440A digitizer. Data were acquired at $5 \mathrm{kHz}$ and low-pass filtered at $2 \mathrm{kHz}$. Cells were held at $-70 \mathrm{mV}$, and cells were discarded if access resistance exceeded $20 \mathrm{M} \Omega$ during recording. Miniature EPSCs (mEPSCs) were identified using a template search followed by visual inspection of each event. For each neuron, the frequency was calculated, and the amplitude was assessed by averaging the amplitude of all events. mEPSC kinetics were determined for each cell by averaging all events aligned to the beginning of the events. Rise times were measured as the time interval between 20 and $80 \%$ of the peak amplitude, and decay time was defined as the interval $\tau$ from 100 to $37 \%$ of the decay phase after fitting. Data analysis was done using pClamp10. Each parameter was plotted as mean \pm SEM for all cells because individual cells are the biggest source of variability in this experiment, and similar numbers of neurons were recorded from each of the three cultures and for each group. Groups were compared by unpaired two-tailed Student's $t$ tests, and the experimenter was blind to the groups throughout data acquisition and analyses.

RNA extraction and $q R T-P C R$. Samples were collected in Trizol (Ambion) and stored at $-80^{\circ} \mathrm{C}$. The RNeasy Mini Kit (Qiagen) was used to extract RNA according to the manufacturer's instructions, including incolumn DNase treatment (Qiagen) cDNA was reverse transcribed using the High Capacity cDNA Reverse Transcription kit (Applied Biosystems). For qPCR, SsoFast Evagreen Supermix (Bio-Rad) was used with the primers in Table 2.

Western blotting. Samples were collected in cold lysis buffer ( $1 \%$ Triton $\mathrm{X}-100$, 50 mм HEPES, pH 7.4, $150 \mathrm{~mm} \mathrm{NaCl}, 1.5 \mathrm{~mm} \mathrm{MgCl}_{2}, 1$ mm EGTA, $10 \%$ glycerol, and freshly added phosphatase inhibitors from Roche Applied Science, catalog \#04906837001). Lysed neurons were mixed 3:1 


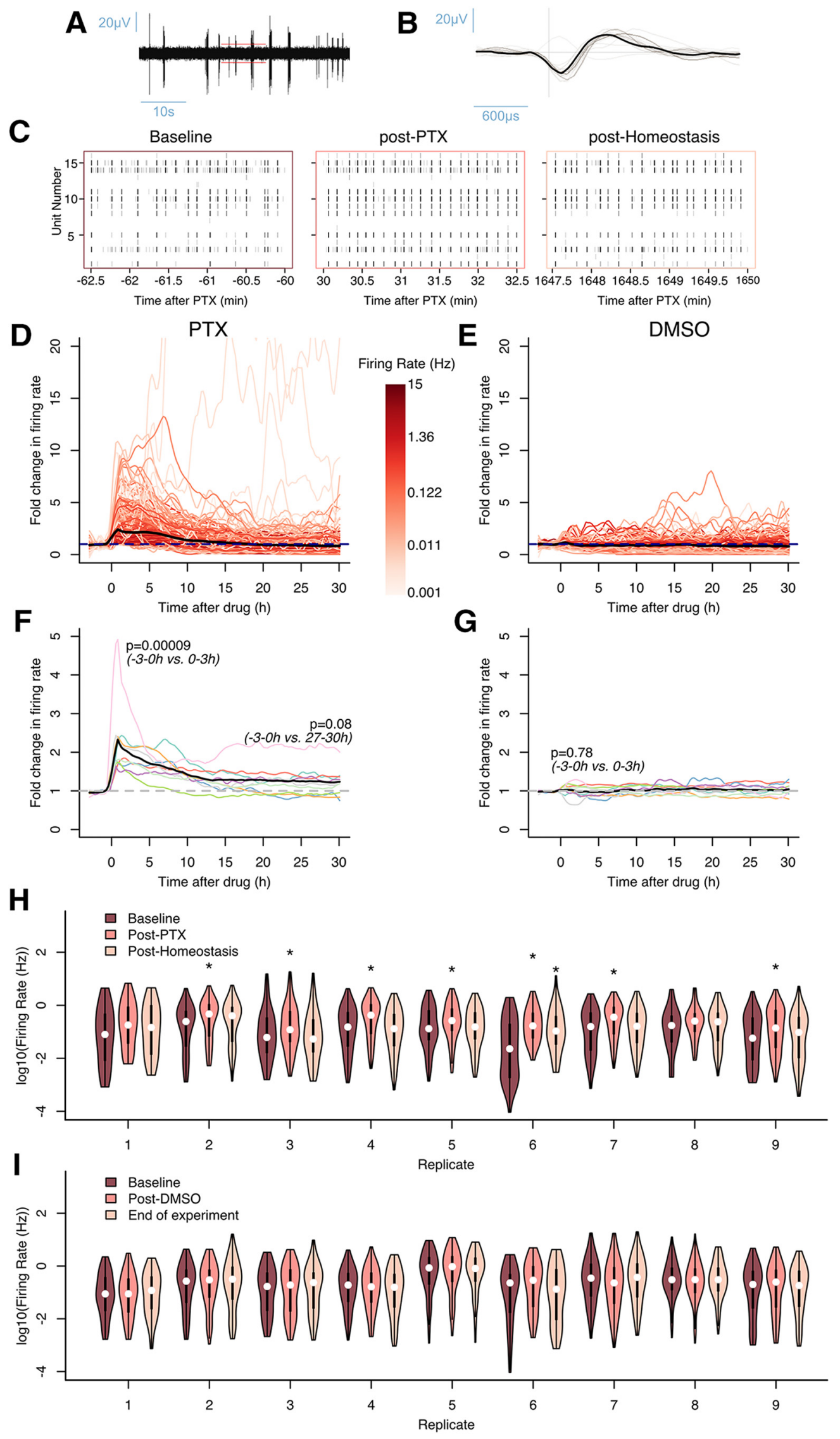

Figure 1. Cultured neurons homeostatically adapt their firing rates in response to PTX treatment. $A$, An example trace of one electrode of an MEA recording from our cultures. The red lines represent the threshold for calling a spike: if the voltage passed 5.5× the SD, it was called as a spike. $\boldsymbol{B}$, An example of the spike waveforms collected from a single (Figure legend continues.) 
Table 2. qPCR primers

\begin{tabular}{lll}
\hline DNA primer name & Sequence & RNA \\
\hline Fos Fw & GGCTCTCCTGTCAACACACA & mRNA \\
Fos Rv & TGTCACCGTGGGATAAAGT & mRNA \\
Npas4 Fw & GGGTGTCTTCTTGGTGGAG & mRNA \\
Npas $4 \mathrm{Rv}$ & CTGCCACAATGTCTTCAAGC & mRNA \\
Pcsk1 Fw & TGCAGGTGAAATGGCATGC & mRNA \\
Pcsk1 Rv & GGCCAGGGTTGAATCCAATTG & mRNA \\
Crem Fw & GCAAAAGCCAACATGGCTG & mRNA \\
Crem Rv & CATGTGACTTGGGGCAGGT & mRNA \\
Arc Fw & GTGAAGACAAGCCAGCATGA & mRNA \\
Arc Rv & CCAAGAGGACCAAGGGTACA & mRNA \\
Gapdh Fw & CGTCCGTAGACAAAATGGT & mRNA \\
Gapdh Rv & TCGTTGATGGCAACAATCTC & mRNA \\
Nptx2 Fw & AGCCAACGAGATTGTGCTGA & mRNA \\
Nptx2 Rv & TCTCGAGTGGTCCAGGTGAT & mRNA \\
\hline
\end{tabular}

with $4 \times$ sample buffer (40\% glycerol, $8 \%$ SDS, 0.25 м Tris- $\mathrm{HCl}, \mathrm{pH} 6.8$, $10 \% 2$-mercaptoethanol) and boiled for $5 \mathrm{~min}$. Samples were centrifuged at full speed for 3 min before loading on NuPage 4-12\% Bis-Tris Gels (Invitrogen). Gels were run at $140 \mathrm{~V}$ for $55 \mathrm{~min}$. We transferred onto nitrocellulose membranes using the Bio-Rad transfer system at $114 \mathrm{~V}$ for $1 \mathrm{~h}$ and $7 \mathrm{~min}$. Membranes were blocked in 5\% milk-Tris-buffered saline + Triton X-100 (TBST) for $1 \mathrm{~h}$. They were treated with primary antibody in $5 \%$ milk-TBST for overnight at $4^{\circ} \mathrm{C}$. To visualize protein, blots were incubated with secondary antibody in TBST in the dark for $45 \mathrm{~min}$. Blots were imaged using a Li-Cor Odyssey. Primary antibodies used include: anti-SRF (Cell Signaling Technology, 5147; RRID:AB_10694554; 1:1000), anti-ARC (Synaptic Systems, 156003; RRID:AB_887694; 1:1000), anti-FOSB (Abcam, 11959; 1:500), anti-JUNB (Cell Signaling Technology, C37F9, catalog \#3753; 1:500), anti-FOS (Abcam, 134122; 1:1000). Secondary antibodies used were as follows: IDR dye 680 goat anti-mouse (Li-Cor; 1:10000), IDR dye 800 goat anti-rabbit (Li-Cor; $1: 10000)$.

Statistical analysis. Statistical analysis was performed in R or Graphpad Prism with the tests listed in the text. For $t$ tests on raw firing rates or on fold-changes, we took the log of the data before performing the tests because these data are log-normally distributed (determined by plotting histograms and $q-q$ plots of firing rates, and supported by previous work (Buzsáki and Mizuseki, 2014). By simulation, we confirmed that median firing rates are normally distributed, and therefore performed a $t$ test without first taking the log of median firing rate data.

Where indicated in the figure legends, $p$ values were FDR-corrected with the Benjamini-Hochberg method using the p.adjust function in R.

$\leftarrow$

(Figure legend continued.) electrode of the MEA. The gray lines represent the waveforms of each spike called. The dark black line represents the average waveform for that electrode. $\boldsymbol{C}$, Raster plots showing all spikes from one representative PTX-treated well from one experiment. Each plot shows a 2.5 min window either before PTX was added (baseline), 30 min after PTX addition (post-PTX) or $>27 \mathrm{~h}$ after PTX addition (post-homeostasis). Each raster line represents a spike. Spikes are plotted as semitransparent lines, so darker-appearing marks represent many spikes occurring close in time. $\boldsymbol{D}$, Representative example of the fold-change in firing rate for one replicate $(n=182$ units). PTX ( $2.5 \mu \mathrm{m}$ ) was added at time 0 . Colored lines are the foldchanges in firing rates of individual neurons, colored by their mean baseline firing rate in hertz. The black line represents the median firing rate for the replicate. $\boldsymbol{E}$, Same as $\boldsymbol{D}$ but DMSO was added at time 0 instead of PTX ( $n=181$ units). $\boldsymbol{F}$, Firing rate homeostasis following PTX stimulation. PTX (2.5 $\mu \mathrm{M})$ was added at time 0 . Colored lines are medians of multiple neurons from each of $n=9$ biological replicates (31-182 U/replicate). The black line represents the mean of these medians. The indicated $p$ values compare the mean of median fold-changes in firing rate between the indicated $3 \mathrm{~h}$ blocks using a two-sided $t$ test on the log(fold-change in firing rate). $\boldsymbol{G}$, Same as $\boldsymbol{F}$ but neurons were treated with a DMSO vehicle control instead of PTX. $\boldsymbol{H}$, Firing rate distributions for each replicate at three time points for PTX-treated units: baseline ( -3 to 0 h), post-PTX ( $0-3 \mathrm{~h}$ ), and post-homeostasis (27-30 h). ${ }^{*} q<0.1$ FDR-adjusted Kolmogorov-Smirnov test comparing indicated distribution to the baseline distribution. $I$, Same as $\boldsymbol{H}$ but with neurons treated with DMSO instead of PTX. $q>0.9$ for all distributions. Data provided in Figure 1-1, available at https://doi.org/10.1523/JNEUROSCI.1108-19.2019.F1-1.
All replicates shown are biological replicates that come from dissection of distinct litters on different days. We noticed that firing rate distributions differed between biological replicates, but were similar within biological replicates over time and across stimulation conditions. Thus, to give lower weight to this replicate-to-replicate variability in initial firing rate distribution (possibly from network formation or plating conditions), we performed paired $t$ tests comparing across stimulation conditions within individual replicates (i.e., pairing different stimulation conditions from the same plate, same recording, and same initial culture).

Binned data from the units used to make plots and perform statistical analysis are available in Figs. 1-1 (available at https://doi.org/10.1523/ JNEUROSCI.1108-19.2019.F1-1); 2-1 (available at https://doi.org/ 10.1523/JNEUROSCI.1108-19.2019.F2-1); 4-1 (available at https:// doi.org/10.1523/JNEUROSCI.1108-19.2019.F4-1); 5-1 (available at https://doi.org/10.1523/JNEUROSCI.1108-19.2019.F5-1); 6-1 (available at https://doi.org/10.1523/JNEUROSCI.1108-19.2019.F6-1); 7-1 (available at https://doi.org/10.1523/JNEUROSCI.1108-19.2019.F7-1).

\section{Results}

\section{Neurons undergo firing rate homeostasis in response to PTX treatment}

To assess firing rate homeostasis, we measured the firing rates of individual neurons over a period of $\sim 30 \mathrm{~h}$ (Fig. $1 A, B$ ) in the presence of a pharmacological perturbation that increased neuronal activity. We cultured dissociated neonatal mouse cortical neurons on MEAs and stimulated them with PTX $(2.5 \mu \mathrm{M})$, which primarily acts as a $\mathrm{GABA}_{\mathrm{A}}$ receptor antagonist, though it also nonspecifically binds to other neural substrates (Johnston et al., 2009). In addition to PTX, we also tested other pharmacological stimuli but found that PTX was the most reliable for inducing increases in firing rate that were followed by firing rate homeostasis (Table 1). To obtain the firing rates of individual units that likely represent single neurons, we spike-sorted the action potential waveforms obtained from MEA recordings (see Materials and Methods). In each experiment, we first recorded baseline neuronal firing rates over a period of $3 \mathrm{~h}$. We then stimulated with PTX, which, in the majority of neurons, induces an increase in firing rate (Fig. $1 C, D, F)$. As we cannot assess firing rate homeostasis in neurons that do not respond to PTX, we focused the rest of our analysis on those that increased their firing rates in response to PTX treatment (see Materials and Methods). Of the neurons affected by PTX stimulation, PTX stimulation resulted in a median 2.78 -fold change in firing rate. Over the next $\sim 10-20 \mathrm{~h}$, the median neuronal firing rate returned to baseline values (Fig. $1 C, D, F)$. Specifically, the firing rate returned to within 1.3 -fold baseline for 7/9 replicates, with the mean of the median firing rates across all replicates not significantly different from baseline in the final $3 \mathrm{~h}$ of the recording (Fig. 1D). This adaptation of median firing rate could occur even if the firing rates of many neurons in the culture remain elevated. We therefore asked whether the distribution of firing rates was altered by PTX treatment. While PTX treatment initially shifts the distribution of firing rates in most replicates $($ Fig. $1 H$ ), the firing rate distribution post-homeostasis (i.e., the last $3 \mathrm{~h}$ of recording) is not significantly different from the distribution during the baseline measurement for all but one replicate (Fig. $1 H$ ), suggesting that the distribution of firing rates in the circuit also homeostatically adapts. Therefore, we observe firing rate homeostasis in response to PTX stimulation.

We next asked whether the apparent firing rate homeostasis that we observed is genuine or due to potential artifacts of the assay. First, we asked whether the increase in neuronal activity that we observed following PTX treatment was because of the drug itself or if it was a side effect of adding PTX (e.g., media 
A

\section{Baseline $\stackrel{3 h}{\rightarrow} \underset{\text { initial stim. wells }}{\text { Add PTX to }} \stackrel{30 h}{\longrightarrow} \begin{gathered}\text { Move PTX } \\ \text { Media }\end{gathered}$}

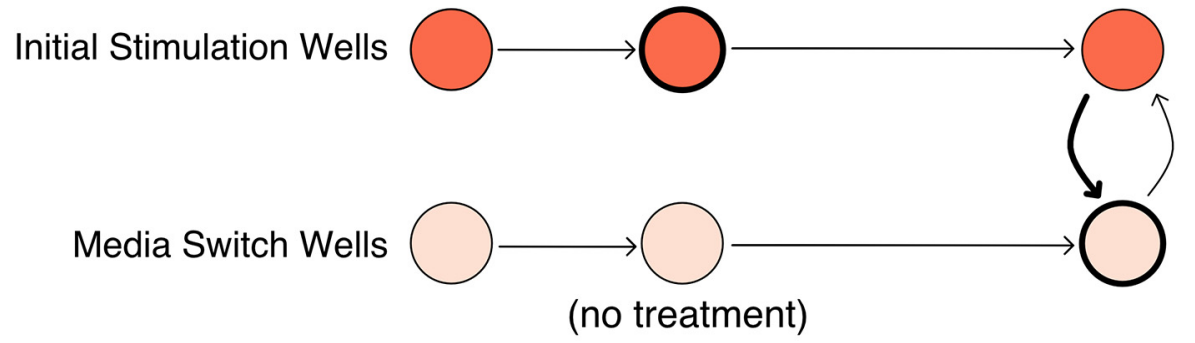

B
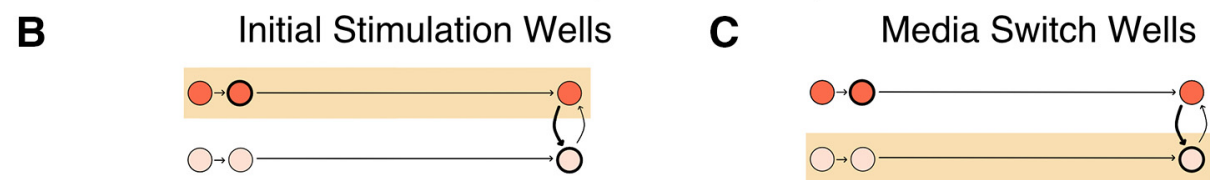

\section{존}

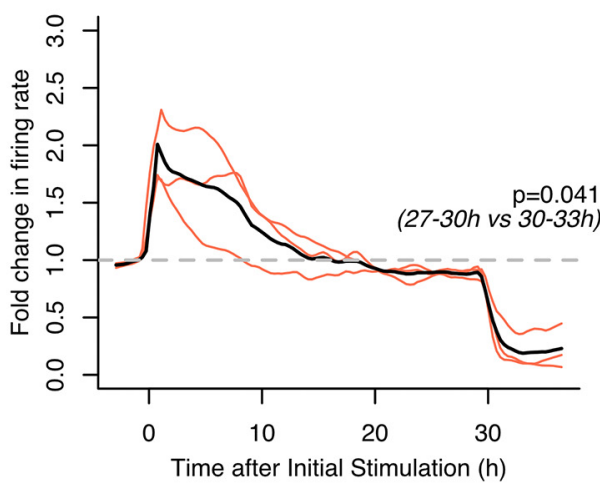

D

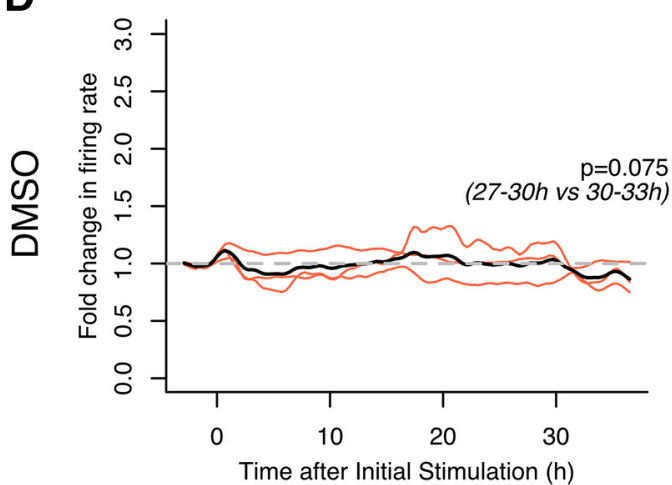

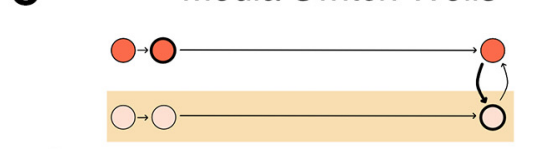
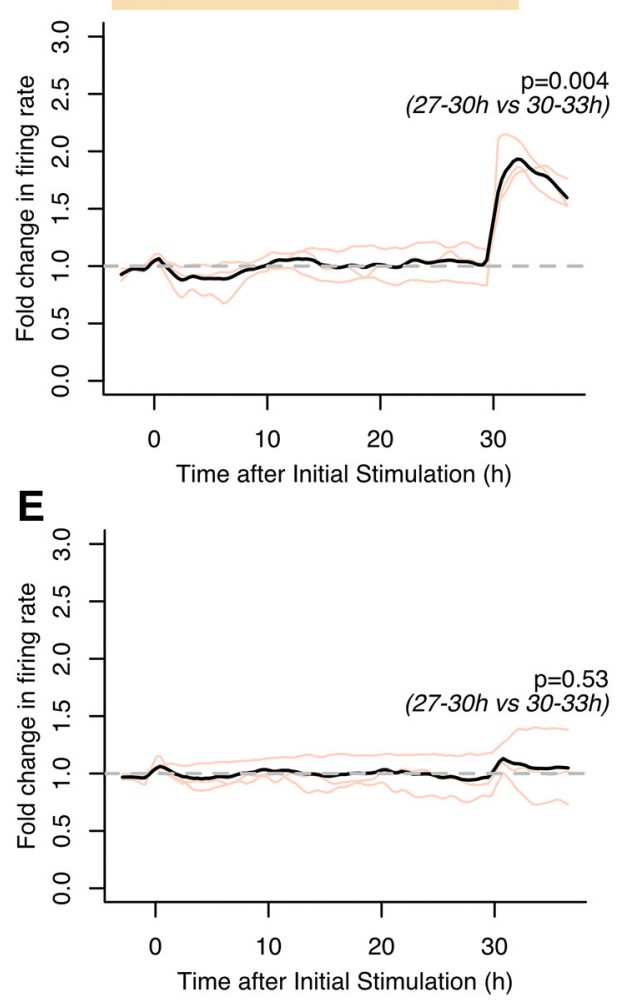

Figure 2. PTX remains potent for the entirety of the homeostasis assay. $A$, Schematic of the media switch experiment to test longevity of PTX potency. One-half of the wells were stimulated with PTX at time $0 \mathrm{~h}$. At time $30 \mathrm{~h}$, the media from the stimulated wells (initial stimulation) was switched with those from unstimulated wells (media switch). $\boldsymbol{B}$, The fold-change in firing rate over the course of the media switch assay in the wells initially stimulated with PTX, $n=3$ biological replicates ( $84-182$ units/replicate). PTX (2.5 $\mu$ m) was added at 0 h and media swapped (i.e., PTX removed) at $30 \mathrm{~h}$. Colored lines are medians from individual replicates. The black line represents the mean of these medians. $P$ value from a two-sided $t$ test on log(median fold-change) testing a difference from a fold-change of 1. C, The fold-change in firing rate over the course of the media switch assay in the wells that received PTX upon media switch, $n=3$ biological replicates (71-159 units/replicate). PTX-containing media from initially stimulated wells was swapped in at $30 \mathrm{~h}$. Colored lines are medians from individual replicates, and the black line is the mean of these medians. $P$ value from a two-sided $t$ test on $\log$ (median fold-change) testing a difference from a fold-change of 1. $\boldsymbol{D}$, Same as $\boldsymbol{B}$ but with DMSO-treated instead of PTX-treated cultures. $\boldsymbol{E}$, Same as $\boldsymbol{C}$ but with DMSO-treated instead of PTX-treated cultures. Data provided in Figure 2-1, available at https://doi.org/10.1523/JNEUROSCI.1108-19.2019.F2-1.

perturbation). We found that neurons treated with a DMSO vehicle do not exhibit an increase in median firing rate or a change in the distribution of firing rates over the $30 \mathrm{~h}$ following addition of the vehicle (Fig. 1E, $G, I$ ), suggesting that the increase in neuronal activity upon stimulation is due to the PTX itself. Next, we asked whether the firing rate homeostasis that we observed could be explained by a decrease in PTX potency over course of the assay. We transferred PTX-containing media from PTX-treated neurons ("initial stimulation wells") to untreated neurons ("media switch wells") at the end of a $30 \mathrm{~h}$ experiment (Fig. 2A). The addition of PTX-containing media increased the firing rates of the previously-untreated neurons to the same extent as the initial PTX treatment [Fig. 2 B, C; fold-change $=1.8$ (initial) vs 2.1 (switch), $p=0.27$ paired $t$ test on median $\log$ (firing rate foldchange)], indicating that the PTX maintains its full potency throughout the experiment. In contrast, neurons newly treated with DMSO-containing media exhibited no such increase in firing rate (Fig. $2 D, E$ ). Finally, we asked whether the apparent firing rate homeostasis we observe is rather because of desensitization (e.g., of the $\mathrm{GABA}_{\mathrm{A}}$ receptor to PTX). If the reduction in firing rate in the continued presence of PTX is due to homeostasis rather than desensitization to PTX, removal of 
PTX should induce an immediate decrease in firing rates. Indeed, we observed a decrease in firing rates upon removal of PTX post-homeostasis (Fig. 2B), suggesting that the firing rate homeostasis we observe results from neurons lowering their overall excitability to adapt to persistent PTX-mediated activation, rather than from desensitization of the $\mathrm{GABA}_{\mathrm{A}}$ receptor or another PTX target. We observed no such decrease in firing rate upon removal of DMSO (Fig. 2D), indicating that the decreased excitability upon PTX withdrawal is not simply due to a change in media. Thus, we are able to measure firing rate homeostasis that occurs via adaptations in excitability in response to persistent neuronal stimulation.

Before assessing the involvement of transcription in firing rate homeostasis, we further characterized several molecular and electrophysiological properties of our assay. First, we asked whether this homeostasis is cell-autonomous, that is, due to individual neurons each adapting to their individual baseline firing rates (Burrone et al., 2002; Hengen et al., 2016; Kulik et al., 2019). Alternatively, the observed firing rate homeostasis could be a non-cell-autonomous, network-level adaptation in which the firing rate of the network adapts homeostatically without firing rate homeostasis of individual neurons (Slomowitz et al., 2015). To distinguish between these possibilities, we compared the firing rates of individual neurons before and after firing rate homeostasis. We found that individual neurons have similar firing rates before and following homeostasis, as demonstrated by their clustering around the unity line in plots of post-homeostasis firing rate against the baseline firing rate (Fig. $3 A$ ). In contrast, poststimulation (i.e., $3 \mathrm{~h}$ after PTX addition), the same neurons have increased firing rates relative to baseline (Fig. 3B). Notably, as observed previously for firing rate homeostasis in response to activity blockade (Slomowitz et al., 2015), a substantial fraction of neurons do not fall exactly on the unity line, indicating that many do not return exactly to their baseline firing rates. However, this "drift" in firing rates over the course of the assay is evident in both PTX-treated neurons, which underwent firing rate homeostasis, and DMSO-treated neurons, which received no stimulation (Figs. $3 A, C$ ). Indeed, the distributions of firing rate drifts between baseline and post-homeostasis were not significantly different between DMSO- and PTX-treated neurons within any of our individual biological replicates (Fig. $3 C$ ), nor in the full dataset (DMSO 0.48 vs PTX 0.60, $p=0.062$, paired twosided $t$ test on medians from each group). This equivalency between DMSO- and PTX-treated neurons indicates that the apparent failure to adapt perfectly is expected based on the observed firing rate drift, which could be because of actual fluctuation or to noise in the measurements of firing rate. In either case, our findings suggest that individual neurons homeostatically maintain their firing rates in the face of excitatory stimulation at least within a range, if not precisely.

Next, if transcription were required for firing rate homeostasis, PTX stimulation should induce ARG transcription. We therefore used qPCR to assess the induction of activity-regulated genes in several mechanistically distinct classes. We chose to assess gene expression at 1 and $6 \mathrm{~h}$ following stimulation to capture both rapidly-induced and delayed genes (Tyssowski et al., 2018). We reasoned that because protein production follows mRNA induction, we would expect the ARG protein products of the genes expressed at $6 \mathrm{~h}$ following PTX treatment to be present in the cell at $8-10 \mathrm{~h}$ after treatment, when firing rates begin to decrease. We selected genes with reported roles in homeostatic plasticity or high fold-induction values in previous work (Tyssowski et al., 2018). We observed induction of the rapid primary response genes Fos, Npas4, and Arc, the delayed primary response genes Pcsk1 and Crem, and the secondary response gene Nptx2 (Fig. 2D). Notably, Arc regulates synaptic scaling up (Shepherd et al., 2006), Npas4 and Nptx2 regulate E/I balance (Chang et al., 2010; Spiegel et al., 2014; Hartzell et al., 2018), and Crem KO mice are severely epileptic, suggesting Crem may also regulate neuronal firing rate homeostasis (Porter et al., 2008). Because we observed transcription of several classes of ARGs, we expect that much of the ARG program, as identified in other experiments (Yap and Greenberg, 2018), is transcriptionally induced in neurons undergoing firing rate homeostasis in response to PTX.

Finally, we asked whether synaptic scaling down occurred in our system, as synaptic scaling in both directions is regulated by ARGs (Shepherd et al., 2006; Ibata et al., 2008; Seeburg et al., 2008; Hu et al., 2010; Diering et al., 2017). Synaptic scaling is defined as a multiplicative change in mEPSC amplitude (Turrigiano et al., 1998). We plated neurons on glass coverslips at the same density as we used on MEAs and treated them with either DMSO or $2.5 \mu \mathrm{M}$ PTX. After 15-20 h of treatment, around the time when neurons return to their baseline firing rates in our MEA experiments, we recorded mEPSCs in the presence of TTX. We observed no difference in average mEPSC amplitudes, frequencies, rise times, or decay times between DMSO-treated and PTX-treated cultures (Fig. 3E-I). Although we cannot rule out very small changes in mEPSC amplitudes in response to PTX treatment, synaptic scaling is unlikely to be a major driver of homeostatic plasticity in our cultures. However, other forms of transcription-dependent homeostasis, namely changes in E/I balance and intrinsic excitability, could still contribute to the firing rate homeostasis we observe.

\section{Firing rate homeostasis occurs in the absence of ARC}

To test the requirement of transcription for firing rate homeostasis, we first focused on the requirement of the ARG Arc, as it has been proposed that activity-dependent $\operatorname{Arc}$ transcription could play a role in homeostatically regulating synaptic metaplasticity (Shepherd and Bear, 2011). To determine whether Arc is required for firing rate homeostasis, we use MEAs to measure the firing rates of cultured neurons from constitutive Arc KO mice (Wang et al., 2006; Fig. 4A) over 30 h of PTX stimulation. In contrast with our prediction that $A r c$ would be important for firing rate homeostasis, we found that by $27 \mathrm{~h}$ of stimulation, the median firing rate and distribution of firing rates of Arc KO neurons return to baseline levels (Fig. $4 B, D$ ), indicating that $A r c \mathrm{KO}$ neurons undergo firing rate homeostasis. We next asked whether Arc $\mathrm{KO}$ might delay firing rate homeostasis. We determined the time at which the median firing rate for each biological replicate returned to baseline levels after PTX addition (see Materials and Methods). This analysis revealed that the time-scale of homeostatic firing rate adaptation in Arc KO neurons is indistinguishable from that in their heterozygous littermates $(15.9 \mathrm{~h}+/-$ vs $16.2 \mathrm{~h}-/-, p=0.96$, two-sided $t$ test), indicating that neurons lacking A $r c$ undergo firing rate homeostasis with normal kinetics. Furthermore, we confirmed that compared with neurons from heterozygote littermates, Arc KO neurons showed similar baseline firing rate medians $(0.34 \mathrm{~Hz}+/-$ vs $0.14 \mathrm{~Hz}-/-, p=0.44$, two-sided paired $t$ test) and distributions (Fig. $4 E$ ). Although we observed a slight difference in baseline firing rate distribution between heterozygous and $A r c \mathrm{KO}$ cultures in one replicate, this was not reproducible. We also confirmed that treatment with a DMSO vehicle in the place of PTX did not perturb firing rates (Fig. 4C), indicating that the observed increases in firing rate are due to PTX treatment. Therefore, Arc KO neurons undergo firing 
A

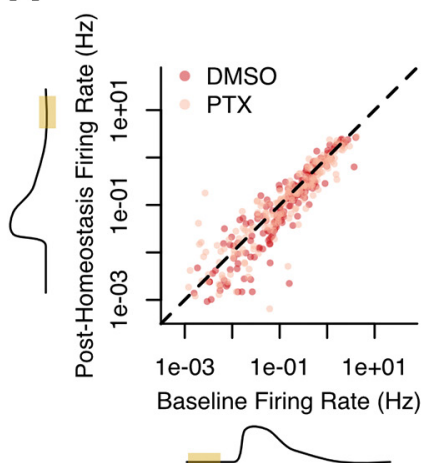

B

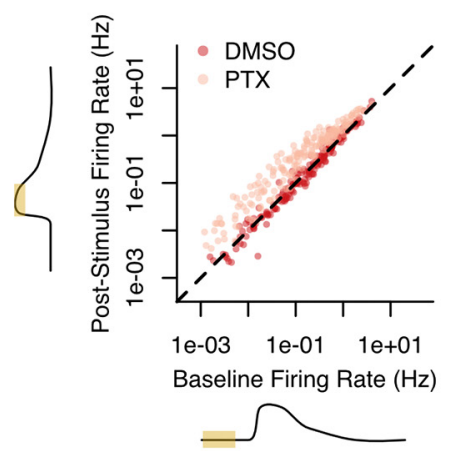

C

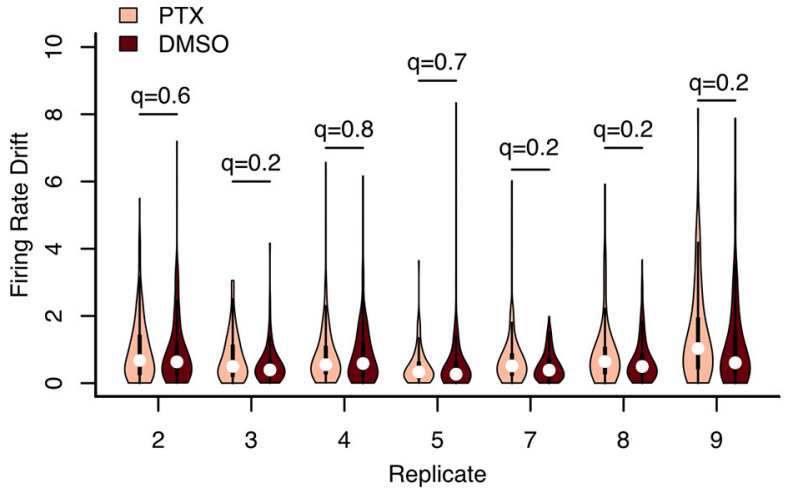

D Fos

Npas4

Arc

Pcsk1
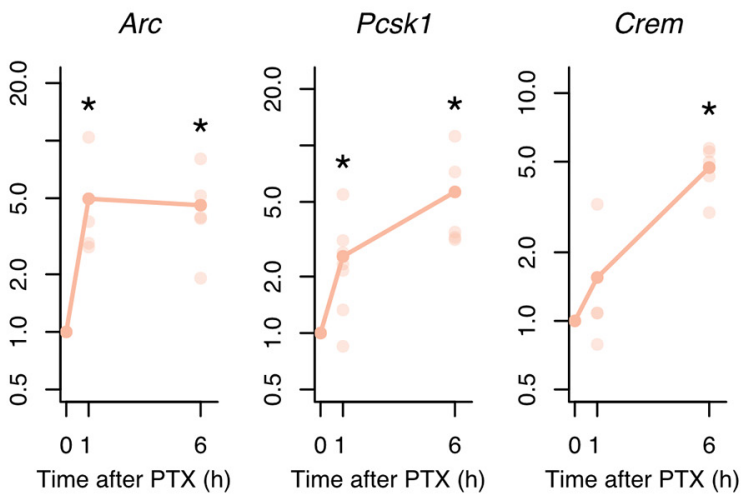

Time after PTX (h)

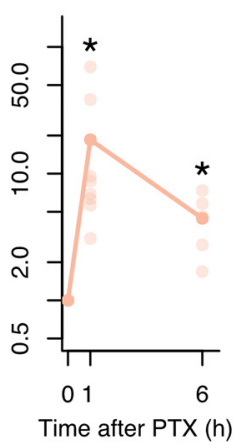

Time after PTX (h)

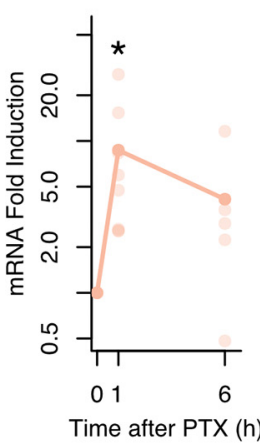

E

\section{DMSO}

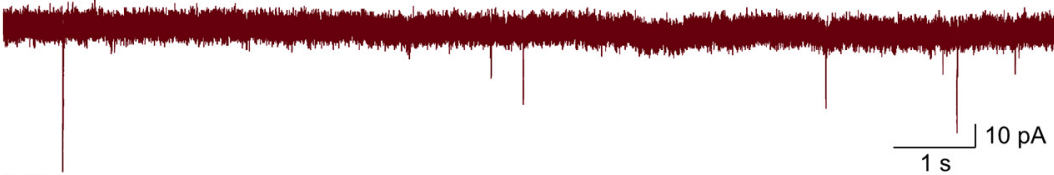

PTX

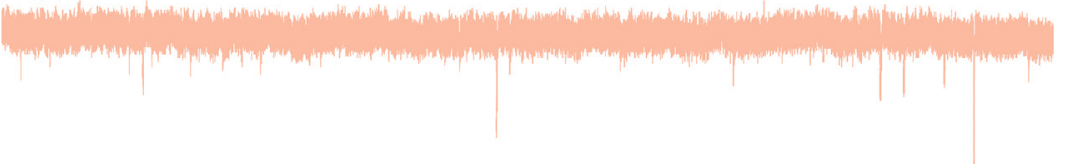

F

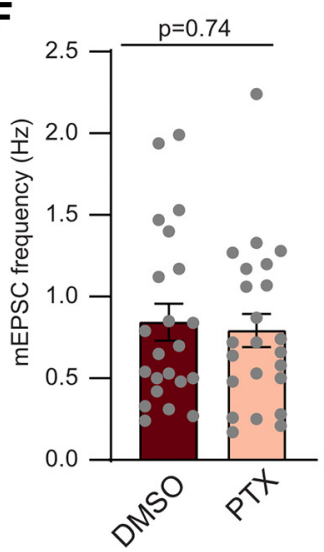

G

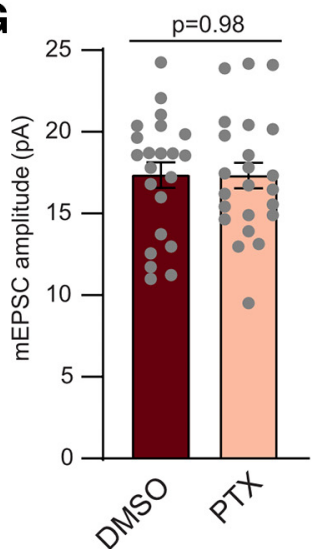

H

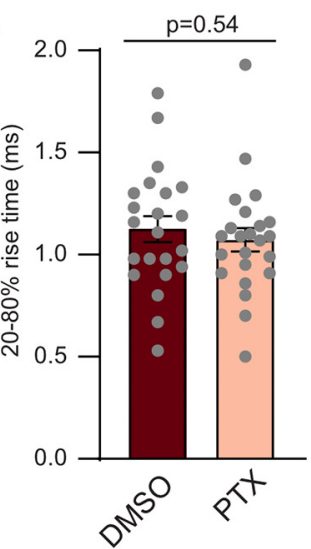

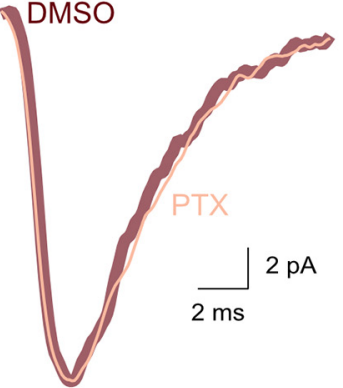

I

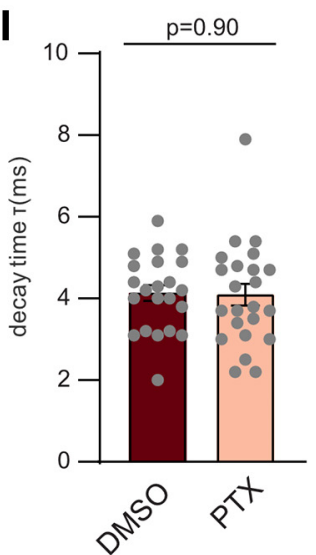

Figure 3. PTX treatment leads to firing rate homeostasis in individual units and induces expression of ARGs in cultured neurons, but does not induce synaptic scaling. $A$, A representative replicate showing a comparison of firing rates before and after homeostasis (baseline, -3 to $0 \mathrm{~h}$; post-homeostasis, 27-30 h). Each point represents an individual unit (units/replicate, DMS0: 181; PTX: 182). $\boldsymbol{B}$, Same as $\boldsymbol{A}$ but comparing firing rates from before stimulation (baseline, -3 to $0 \mathrm{~h}$ ) to soon after addition of PTX or DMSO (poststimulation, $0.5-3.5 \mathrm{~h}$ ). $\boldsymbol{C}$, Comparison of firing rate drift distributions at the end of the assay (27-30 h) in DMSO-treated and PTX-treated cultures. Firing rate drift was calculated as the |log2(post-homeostasis/baseline) to weight changes from a fold-change of 1 equally. $n=7$ biological replicates (units/replicate: DMS0, 87-187; PTX, 84-182), 2 replicates from Figure 1 ( 1 and \#) were eliminated because they failed to undergo firing rate homeostasis (median firing rate in last $3 \mathrm{~h}>1.5$-fold different from median in first $3 \mathrm{~h}$ ). Median drift is indistinguishable between PTX and DMSO treatments in all replicates: $q$ values are FDR-adjusted $p$ values from rank sum tests comparing drifts in PTX- and DMSO-treated cultures. D, PTX stimulation induces mRNA expression. Fold (Figure legend continues.) 
A

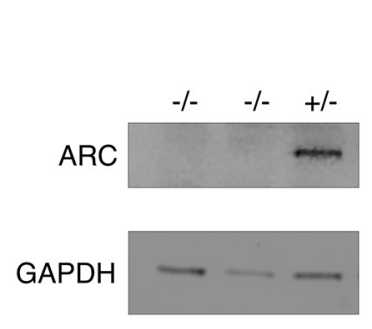

B

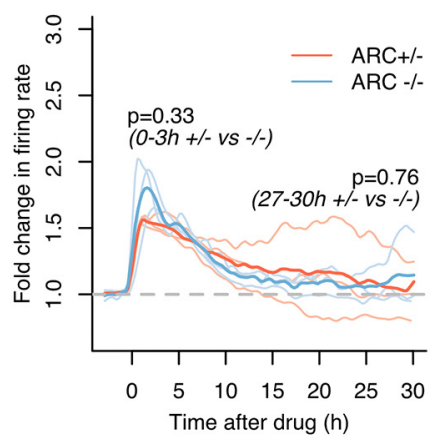

D

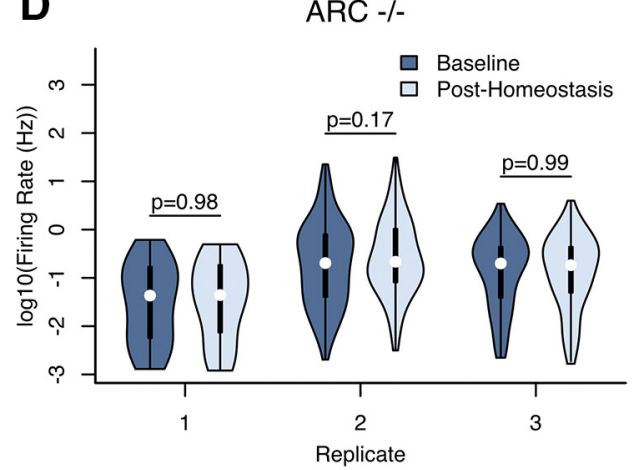

C

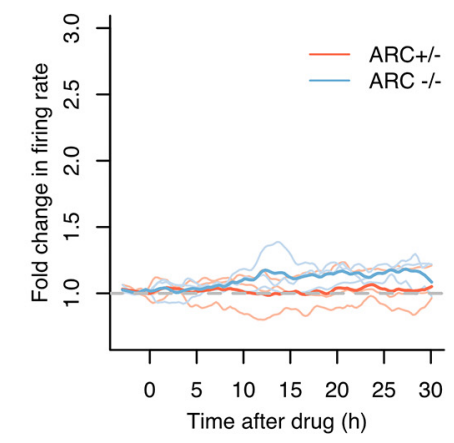

DMSO-treated
$\mathbf{E}$

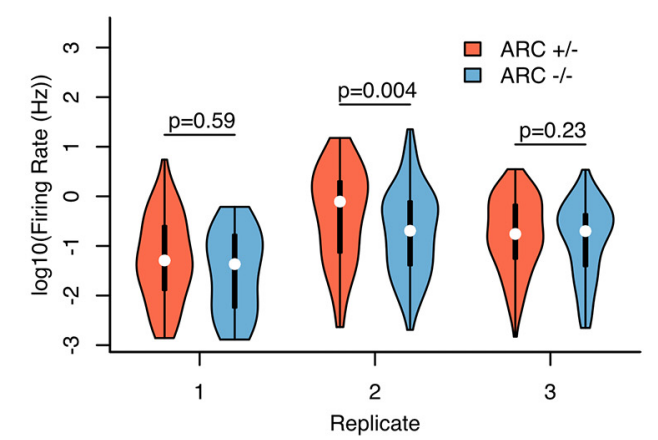

Figure 4. Firing rate homeostasis occurs in the absence of ARC. $A$, Confirmation of ARC KO. Cultured neurons were stimulated with potassium chloride for $2 \mathrm{~h}$ to induce ARC protein before collecting. A representative image from one of $n=3$ biological replicates. $\boldsymbol{B}$, The fold-change in firing rate over the course of the assay. PTX ( $2.5 \mu \mathrm{m}$ ) was added at time 0 . Lighter lines are medians from each of $n=3$ biological replicates (units/replicate: Arc $+/-, 64-135 ;$ Arc $-1-, 20-128)$. Darker lines represent the mean of the median firing rates for replicates from each genotype. $\operatorname{Arc}-1-$ return to their baseline firing rates by the end of the assay: $p=0.46, t$ test of log fold-change in firing rates between baseline and the last $3 \mathrm{~h}$ of the assay $(-3$ to $0 \mathrm{~h}, \mathrm{vs} 27-30 \mathrm{~h})$, testing difference from fold-change $=1$. $P$ values shown represent a $t$ test on log (fold-change in firing rate) comparing ARC $+/-$ and ARC $-I-$ cultures. $C$, The fold-change in firing rate over the course of the assay. DMSO was added at time 0 . Lighter lines are medians from each of $n=3$ biological replicates (units/replicate: $\operatorname{Arc}+/-, 99-215 ; \operatorname{Arc}-/-, 40-291$ ). Darker lines represent the mean of the median firing rates for replicates from each genotype. $D$, Firing rate distributions for each replicate at two time points for PTX-treated Arc $-/-$ units: baseline ( -3 to $0 \mathrm{~h}$ ) and posthomeostasis (27-30 h). $q$ values represent FDR-adjusted Kolmogorov-Smirnov test comparing indicated distributions. E, Baseline firing rate ( -3 to 0 h) distributions for PTX-treated units comparing ARC $-1-$ and ARC $+1-$ cultures. $q$ values represent FDR-adjusted Kolmogorov-Smirnov test comparing indicated distributions. Data provided in Figure 4-1, available at https://doi.org/10.1523/JNEUROSCI.1108-19.2019.F4-1.

rate homeostasis that is indistinguishable, both in extent of adaptation and kinetics, from that of control neurons, indicating that firing rate homeostasis still occurs in the absence of Arc.

Firing rate homeostasis occurs in the absence of SRF and AP1 We next hypothesized that other ARGs may compensate for the loss of $A r c$, or be independently required, in regulating firing rate homeostasis. We therefore aimed to simultaneously block the activity-regulated transcription of many ARGs by manipulating activity-regulated transcription factors. We focused on two transcription factors. First, we knocked out SRF, which is required for induction of a large subset of ARGs (Ramanan et al., 2005; Kuzniewska et al., 2016; Lösing et al., 2017). Second, we knocked out three inducible components of the AP1 transcription factor complex, which regulates slowly-induced ARGs (Vierbuchen et

\section{$\leftarrow$}

(Figure legend continued.) induction calculated from GAPDH-normalized values from RTqPCR. Each lighter dot represents the mRNA expression of a single replicate, and the darker dots show the mean expression. ${ }^{*} p<0.05 t$ test on log2(fold-change), FDR $<0.1$. ( $n=3-8$ biological replicates). $\boldsymbol{E}$, Representative recordings of $\mathrm{mEPSCS}$ (left) and the averaged mEPSC from a single neuron (right) in PTX- or DMSO-treated neurons. $\boldsymbol{F}-\boldsymbol{I}$, Analysis of mEPSC frequency $(\boldsymbol{F})$, amplitude $(\boldsymbol{G}), 20 \%-80 \%$ rise time $(\boldsymbol{H})$, and decay time $\tau(\boldsymbol{I})$ in PTXor DMSO-treated cultures (DMSO, $n=22$ cells $/ 3$ independent cultures; PTX, $n=23 / 3, p$ values from Student's $t$ tests). Data for $\boldsymbol{A}-\boldsymbol{C}$ provided in Figure 1-1, available at https:// doi.org/10.1523/JNEUROSCI.1108-19.2019.F1-1. al., 2017; Yap and Greenberg, 2018). Genes regulated by AP1 include Nptx2 and Igf1, which regulate excitatory-inhibitory balance (Chang et al., 2010; Malik et al., 2014; Mardinly et al., 2016). We dissected cortical neurons from either an SRF cKO mouse line homozygous for floxed SRF (Ramanan et al., 2005) or an AP1 triple cKO mouse line with floxed alleles of the AP1 subunits Fos, Fosb, and Junb (Vierbuchen et al., 2017). We treated neurons from each mouse line with AAV-CaMKII-Cre to KO SRF or AP1 in excitatory neurons and confirmed conditional KO by Western blot (Figs. 5A, 6A). In all analyses, we compared Cre-infected neurons to neurons of the same genotype infected with AAV-CaMKII-GFP.

To determine whether SRF or AP1 are required for firing rate homeostasis, we measured the firing rates of SRF cKO and AP1 cKO neurons in response to $30 \mathrm{~h}$ of PTX stimulation. By $27 \mathrm{~h}$ of stimulation, the median firing rate and distribution of firing rates of Cre-treated SRF cKO neurons return to baseline levels (Fig. $5 B, D)$, indicating that neurons lacking SRF still undergo firing rate homeostasis. Median firing rates of neurons lacking AP1 returned to levels slightly above baseline $(<1.5 \times$ baseline), but were not significantly different from the control-treated neurons from the same experiments, suggesting that AP1 cKO does not impair firing rate homeostasis (Fig. 6B). Consistent with this conclusion, baseline firing rate distributions of Cre-treated AP1 cKO neurons were indistinguishable from firing rate distributions at 
A

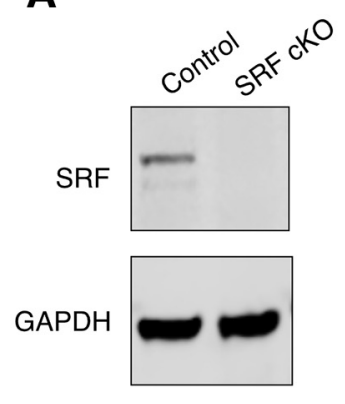

B

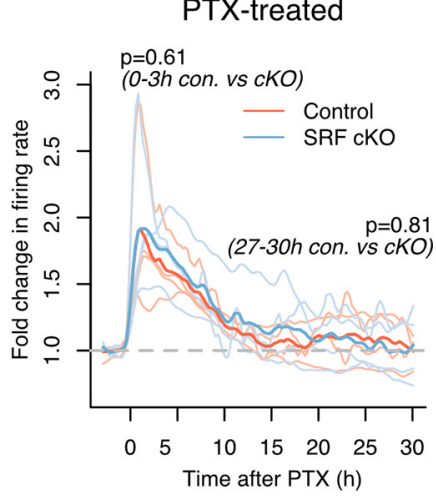

C

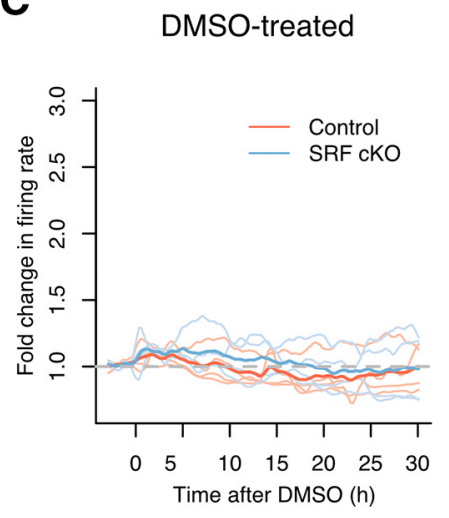

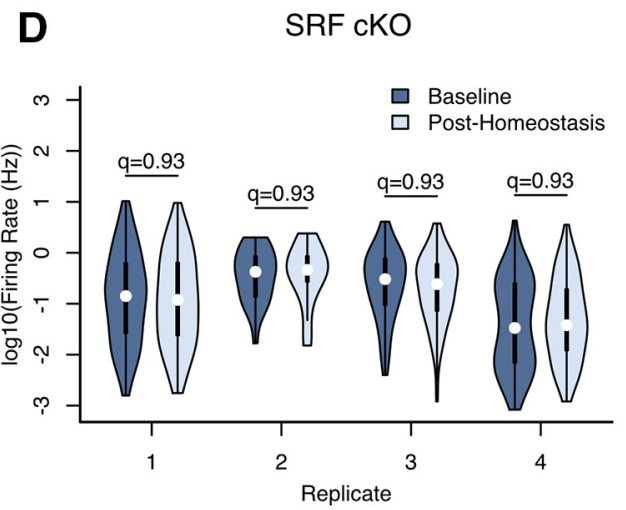

E

Baseline Firing Rates

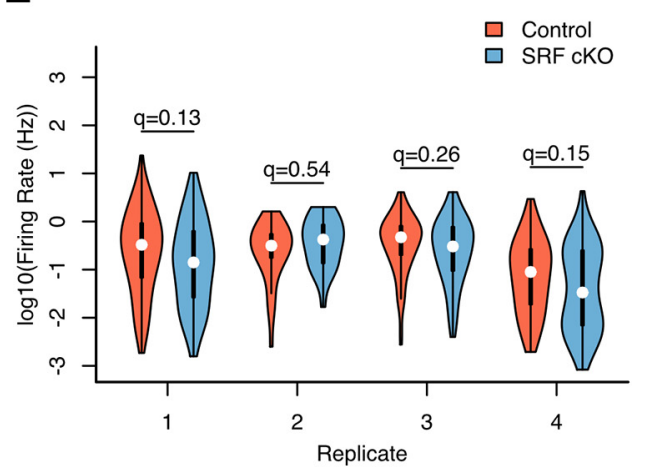

Figure 5. Firing rate homeostasis occurs in the absence of SRF. A, Confirmation of SRF KO in cultured neurons. A representative Western blot image from one of $n=3$ biological replicates. $B$, The fold-change in firing rate over the course of the assay. PTX (2.5 $\mu \mathrm{M}$ ) was added at time 0 . Lighter lines are medians from each of $n=4$ biological replicates (units/replicate: Control, 32-113; SRF K0, $26-174)$. Darker lines represent the mean of the median firing rates for replicates from each genotype. SRF cK0 adapt back to their baseline firing rates by the end of the assay: $p=0.99, t$ test of $\log$ fold-change in firing rates between baseline and the last $3 \mathrm{~h}$ of the assay $(-3$ to $0 \mathrm{~h}$, vs $27-30 \mathrm{~h})$, testing the difference from fold-change $=1$. $P$ values shown from a t test on log $($ fold-change in firing rate) comparing control and cKO cultures. C, The fold-change in firing rate over the course of the assay. DMSO was added at time 0 . Lighter lines are medians from each of $n=4$ biological replicates (units/replicate: Control, 152-295; SRF K0, 130-276). Darker lines represent the mean of the median firing rates for replicates from each genotype. $\boldsymbol{D}$, Firing rate distributions for each replicate at two time points for PTX-treated units: baseline ( -3 to $0 \mathrm{~h}$ ) and post-homeostasis ( $27-30 \mathrm{~h}) . q$ values represent FDR-adjusted Kolmogorov-Smirnov test comparing indicated distributions. $E$, Baseline firing rate ( -3 to $0 \mathrm{~h}$ ) distributions for PTX-treated units comparing control and cKO cultures. $q$ values represent FDR-adjusted Kolmogorov-Smirnov test comparing indicated distributions. Data provided in Figure 5-1, available at https://doi.org/10.1523/JNEUROSCI.1108-19.2019.F5-1.

the end of the assay (Fig. 6D). Furthermore, the time-scale of homeostatic firing rate adaptation in Cre-treated SRF and AP1 cKO neurons is indistinguishable from that of GFP-treated neurons (SRF: $13.0 \mathrm{~h}$ control vs $14.9 \mathrm{~h}$ cKO, $p=0.60$, two-sided $t$ test; AP1: $9.0 \mathrm{~h}$ control vs $8.2 \mathrm{~h} c K O, p=0.77$, two-sided $t$ test), suggesting that neurons lacking AP1 or SRF undergo firing rate homeostasis with normal kinetics. However, as we knocked out AP1 and SRF only in excitatory neurons, we cannot rule out the possibility that their activity in inhibitory neurons mediates firing rate homeostasis. For both genotypes, Cre- and GFP-infected neurons have indistinguishable baseline firing rate distributions (Figs. $5 E, 6 E$ ), and indistinguishable median firing rates (SRF, average median $=0.30 \mathrm{~Hz}$ control vs $0.23 \mathrm{~Hz} \mathrm{cKO}, p=0.35$, two-sided paired $t$ test; AP 1 , average median $=0.20 \mathrm{~Hz}$ control vs $0.13 \mathrm{~Hz}$ cKO, $p=0.34$, two-sided paired $t$ test). Furthermore, treatment of Cre-infected neurons with a DMSO vehicle in the place of PTX did not perturb firing rates (Figs. $5 C, 6 C$ ), indicating that the observed increases in firing rate are due to PTX treatment. Therefore, SRF KO and AP1 KO neurons undergo firing rate homeostasis that is indistinguishable, both in extent of adaptation and kinetics, from that of control neurons, indicating that firing rate homeostasis can still occur in the absence of SRF or AP1, at least in excitatory neurons.
Firing rate homeostasis occurs in the absence of transcription We next sought to entirely block ARG induction to rule out the possibility that ARGs compensate for each other in regulating homeostasis. To acutely block all ARG transcription, we added two different transcription inhibitors (ActD and FLA) to wildtype neurons $30 \mathrm{~min}$ before PTX stimulation and confirmed that they blocked transcription for the duration of the experiment (Fig. 7A,B). To determine whether neurons can undergo firing rate homeostasis in the absence of transcription, we observed the firing rates of ActD-treated and FLA-treated neurons in response to $30 \mathrm{~h}$ of PTX stimulation. As we observed with ARC, SRF, and $\mathrm{AP} 1 \mathrm{KO}$ neurons, by $27 \mathrm{~h}$ of stimulation, the median firing rates and distributions of firing rates of and FLA-treated and ActDtreated neurons return approximately to baseline levels (Fig. 7C$F)$. Furthermore, the time-scale of homeostatic firing rate adaptation ActD- and FLA-treated is indistinguishable from that of untreated neurons measured in the same experiment $(14.6 \mathrm{~h}$ ActD vs $16.8 \mathrm{~h}$ control, $p=0.54 ; 7.1 \mathrm{~h}$ FLA vs $9.3 \mathrm{~h}$ con, $p=0.68$, two-sided $t$ test). Neurons treated with ActD or FLA showed no difference in their response to PTX (Fig. 7C, D), indicating similar sensitivity to PTX. However, ActD-treated neurons stimulated with a DMSO vehicle in the place of PTX showed a rapid decrease in firing rate over the first $5-10 \mathrm{~h}$ following ActD treat- 
A

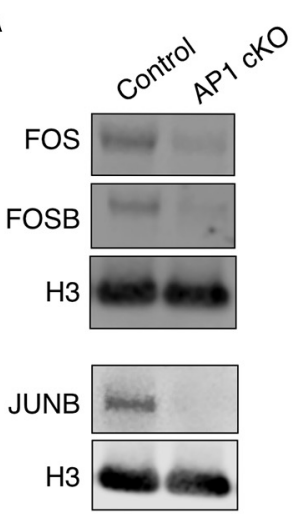

B

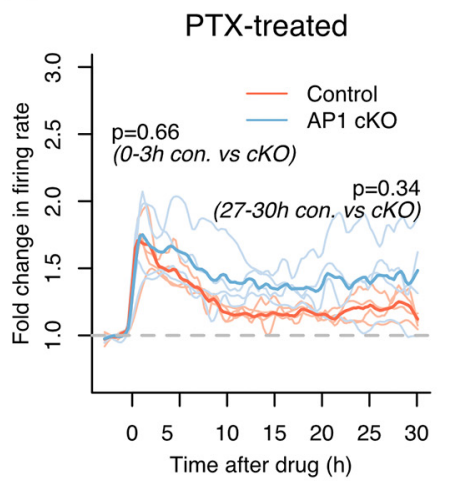

C

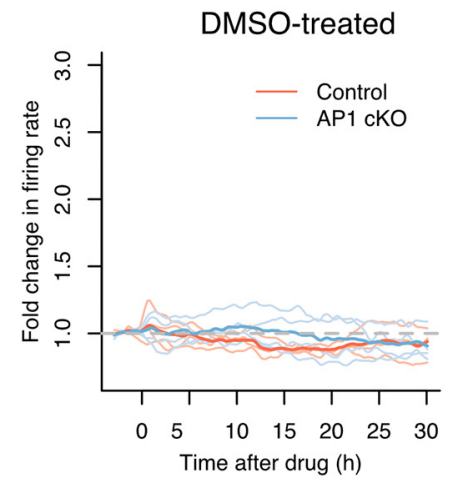

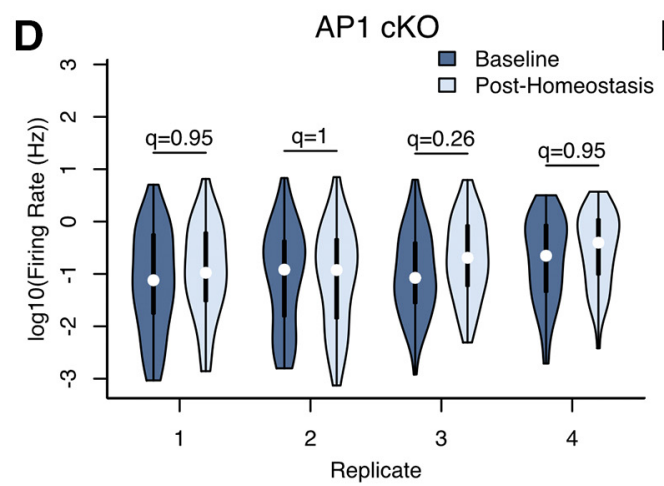

E

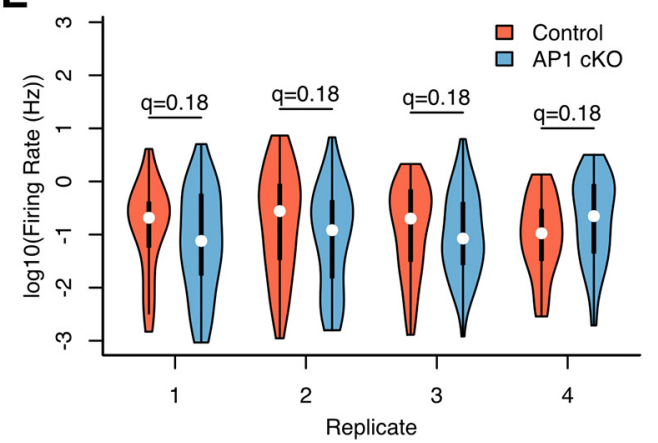

Figure 6. Firing rate homeostasis occurs in the absence of AP1. $A$, Western blot confirmation of AP1 cK0 in cultured neurons. FOS, FOSB, and JUNB are the three floxed AP1 subunits. A representative image from one of $n=3$ biological replicates. $\boldsymbol{B}$, The fold-change in firing rate over the course of the assay. PTX $(2.5 \mu \mathrm{m})$ was added at time 0 . Lighter lines are medians from each of $n=4$ biological replicates (units/replicate: Control, 49-96; AP1 cK0: 44-84). Darker lines represent the mean of the median firing rates for replicates from each genotype. AP1 cK0 and control neurons are slightly higher than baseline by the end of the assay: $p>0.05, t$ test of log fold-change in firing rates between baseline and the last $3 \mathrm{~h}$ of the assay $(-3$ to $0 \mathrm{~h}$, vs $27-30 \mathrm{~h}$ ), testing the difference from fold-change $=1$. $P$ values shown from a t test on $\log$ (fold-change in firing rate) comparing control and cKO cultures. $C$, The fold-change in firing rate over the course of the assay. DMSO was added at time 0 . Lighter lines are medians from each of $n=4$ biological replicates (units/replicate: Control, 81-205; AP1 cK0: 105-195). Darker lines represent the mean of the median firing rates for replicates from each genotype. $\boldsymbol{D}$, Firing rate distributions for each replicate at two time points for PTX-treated units: baseline $(-3$ to $0 \mathrm{~h})$ and post-homeostasis $(27-30 \mathrm{~h})$. $q$ values represent FDR-adjusted Kolmogorov-Smirnov test comparing indicated distributions. $\boldsymbol{E}$, Baseline firing rate ( -3 to $0 \mathrm{~h}$ ) distributions for PTX-treated units comparing control and cK0 cultures. 9 values represent FDR-adjusted Kolmogorov-Smirnov test comparing indicated distributions. Data provided in Figure 6-1, available at https://doi.org/10.1523/JNEUROSCI.1108-19.2019.F6-1.

ment (Fig. 7G), indicating that ActD itself affects firing rate. Indeed, we observed that the that post-homeostasis firing rates of PTX- and ActD-treated neurons were slightly below baseline, consistent with this ActD side effect (Fig. 7E, $H$ ). This consistency between post-homeostasis firing rates in PTX-treated neurons and firing rates at the end of the assay in DMSO-treated neurons suggests that the ActD-treatment side effect does not impair our ability to measure firing rate homeostasis in ActD-treated neurons, rather, it changes the baseline to which neurons adapt. We observed no such change in neuronal firing in DMSO-treated neurons exposed to FLA, suggesting that FLA does not impair firing rate on its own (Fig. 7I). The observation of firing rate homeostasis in the presence of acute total blockades of transcription, along with our findings in AP1, SRF, and Arc KO neurons, indicates that firing rate homeostasis can occur in the absence of activity-regulated transcription.

\section{Discussion}

Our data indicate that neurons undergo firing rate homeostasis in the absence of activity-regulated transcription. Specifically, cultured cortical neurons from $\mathrm{KO}$ mice lacking the ARG Arc, or those without the activity-regulated transcription factors AP1 or SRF, homeostatically adapt their firing rates in the presence of prolonged PTX stimulation. We further found that neurons also undergo firing rate homeostasis in the presence of an acute blockade of transcription with ActD or FLA.
These findings demonstrate that despite the reported roles of activity-regulated transcription in several forms of homeostatic plasticity, neuronal networks can homeostatically reduce their firing rates in response to increases in circuit activity without activity-regulated transcription.

\section{Mechanisms underlying firing rate homeostasis}

Our finding that neurons undergo firing rate homeostasis in the absence of transcription raises the question of how firing rate homeostasis is regulated. We achieved increases in activity via PTX treatment, which is known to block $\mathrm{GABA}_{\mathrm{A}}$ receptors. In other studies, $\mathrm{GABA}_{\mathrm{A}}$ blockade induces synaptic scaling down and homeostatic decreases in intrinsic excitability (Turrigiano et al., 1998; Lee and Chung, 2014). Previous work suggests that both of these forms of homeostasis may be mediated by activityregulated transcription (Shepherd et al., 2006; Goold and Nicoll, 2010; O'Leary et al., 2014; Cho et al., 2016; Diering et al., 2017; Kulik et al., 2019). In our cultures, we were unable to observe synaptic scaling down in response to PTX treatment. This could be because of the low concentration of PTX (up to 40 times lower) that we used relative to other studies that have observed synaptic scaling down in response to PTX treatment (Benevento et al., 2016; Mao et al., 2018). Neurons may require a greater change in activity to trigger synaptic scaling down, which may be achieved with a higher concentration of PTX. Furthermore, this lower concentration could explain why not all neurons in a cul- 


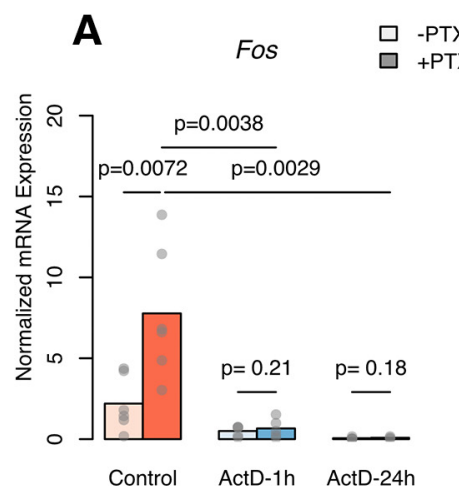

C PTX-treated

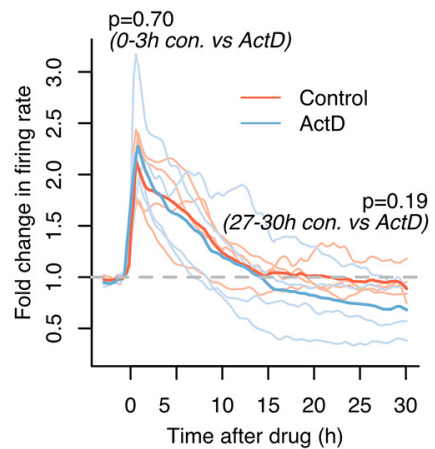

E

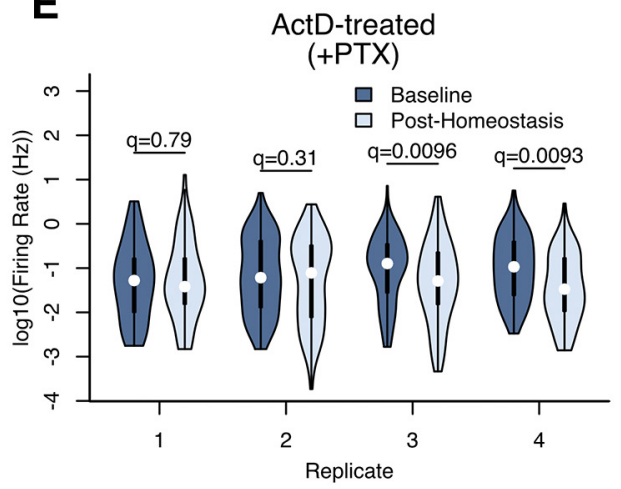

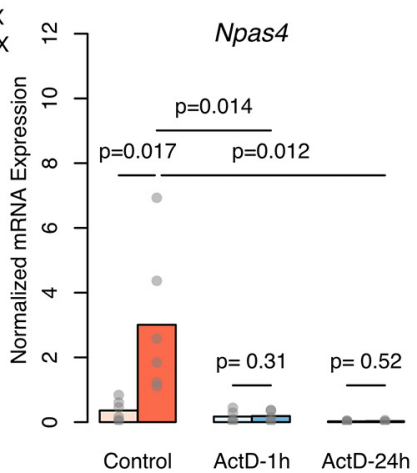

B

pre-Gapdh

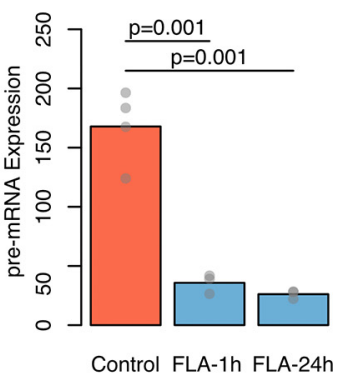

\section{PTX-treated}

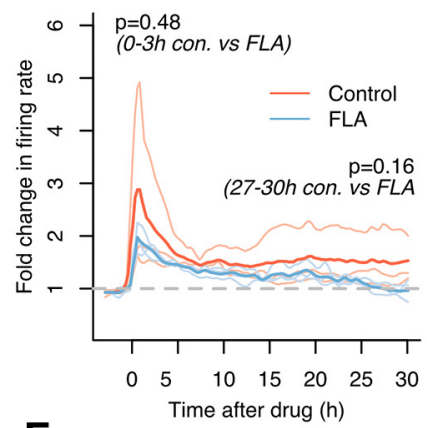

$\mathbf{F}$

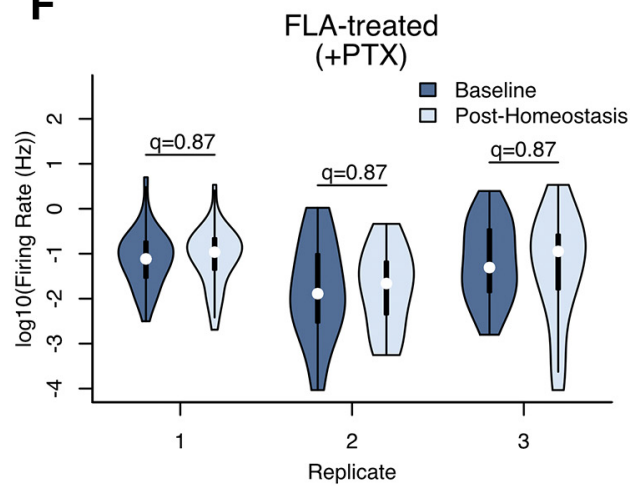

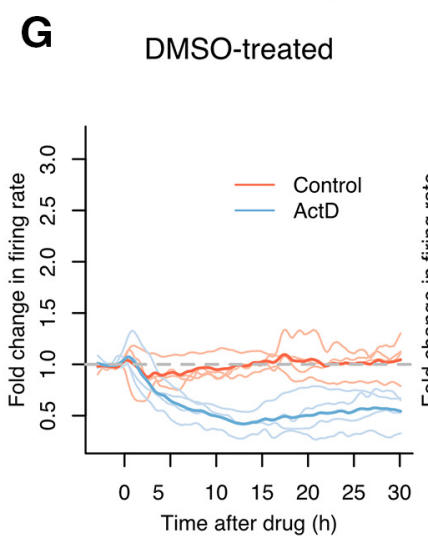

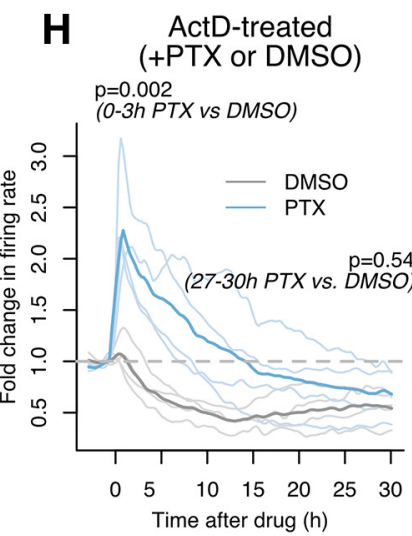

I DMSO-treated

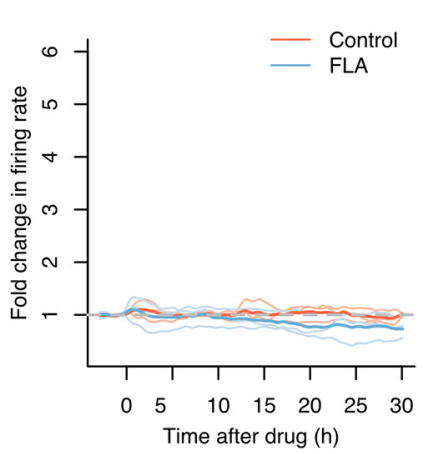

Figure 7. Neurons undergo firing rate homeostasis in the presence of acute transcription blockade. A, ActD treatment blocks activity-regulated transcription. ActD-1 $\mathrm{h}$ samples were treated with ActD for $1.5 \mathrm{~h}$ and stimulated with PTX for $1 \mathrm{~h}(n=6)$. ActD-24 $\mathrm{h}$ samples were treated with ActD for $24.5 \mathrm{~h}$ and stimulated with PTX for the last $1 \mathrm{~h}$ of ActD treatment $(n=3)$. Gapdh-normalized values from RT-qPCR. Dots represent gene expression in each biological replicate, and bars show the mean expression levels. $P$ values from paired, one-sided $t$ tests. $\boldsymbol{B}$, FLA treatment blocks transcription. FLA-1 h samples were treated with FLA for $1 \mathrm{~h}$, and FLA-24 h samples were treated with FLA for $24 \mathrm{~h}$. As a measure of transcription, Gapdh pre-mRNA levels were assessed by RT-qPCR with equal RNA inputs to the RT reaction in $n=3-4$ biological replicates. Dots represent gene expression in each biological replicate, and bars show the mean expression levels. $P$ values from paired, one-sided $t$ tests. $C$, The fold-change in firing rate over the course of the assay with ActD treatment. PTX ( $2.5 \mu \mathrm{m})$ was added at time 0 . Lighter lines are medians from each of $n=4$ biological replicates (units/replicate: Control, 84-182; ActD, 58-169). Darker lines represent the mean of the median firing rates for replicates from each treatment $(n=4)$ ). ActD-treated neurons adapt back to their baseline firing rates by the end of the assay: $p=0.15, t$ test of log fold-change in firing rates between the baseline and the last $3 \mathrm{~h}$ of the assay (Figure legend continues.) 
ture increased their firing in response to PTX treatment. Thus, we cannot rule out the possibility that the low concentration of PTX induced a very modest synaptic scaling down, which we were unable to detect. However, the fact that we observed robust firing rate homeostasis but not synaptic scaling in response to PTX treatment suggests that firing rate homeostasis can occur in the absence of synaptic scaling.

$\mathrm{GABA}_{\mathrm{A}}$ blockade also induces homeostatic decreases in intrinsic excitability (Lee and Chung, 2014), and we therefore suspect that changes in intrinsic excitability could underlie the firing rate homeostasis observed here. It has been proposed that ion channel genes (e.g., potassium channels) could be transcriptionally induced in response to increases in activity and act to homeostatically decrease intrinsic excitability and thus firing rates (O'Leary et al., 2014; Cho et al., 2016; Kulik et al., 2019). However, no studies have directly linked activity-dependent transcriptional regulation of potassium channels to homeostatic regulation of intrinsic excitability. Therefore, activity-regulatedtranscription-independent potassium channel regulation, such as translocation to the membrane, could underlie the firing rate homeostasis that we observe. In addition to regulation of potassium channels, neurons also decrease their intrinsic excitability by moving their axon initial segments further from the cell body (Grubb and Burrone, 2010; Kuba et al., 2010), a process that has not been shown to require activity-regulated transcription. Thus, regulation of intrinsic excitability, either via changes in ion channel use or in axon initial segment position, remains a plausible underlying mechanism of firing rate homeostasis in response to persistent PTX treatment.

We also considered whether several other forms of homeostatic plasticity that have not yet been demonstrated to occur following $\mathrm{GABA}_{\mathrm{A}}$ blockade might underlie the firing rate homeostasis we observe. One such mechanism is alteration in E/I balance, the number of excitatory relative to inhibitory synapses. Our manipulations of activity-regulated transcription likely prevent some alterations in E/I balance: homeostatic decreases in excitatory synaptic input onto excitatory neurons (Goold and Nicoll, 2010) are blocked by acute blockade of transcription, and KO experiments have demonstrated that homeostatic changes in inhibitory synapse number onto specific neuronal subtypes requires several individual ARGs (Bloodgood et al., 2013; Spiegel et

\footnotetext{
$\leftarrow$

(Figure legend continued.) $\quad(-3$ to $0 \mathrm{~h}$, vs $27-30 \mathrm{~h})$, testing difference from fold-change $=1$. $P$ values shown from a $t$ test on log (fold-change in firing rate) comparing control and inhibitortreated cultures. $\boldsymbol{D}$, Like $(\boldsymbol{C})$ but with FLA treatment. $n=3$ biological replicates (units/replicate: Control, 61-116; FLA, 22-73). FLA-treated neurons adapt back to their baseline firing rates by the end of the assay: $p=0.31, t$ test of log fold-change in firing rates between the baseline and the last $3 \mathrm{~h}$ of the assay ( -3 to $0 \mathrm{~h}$, vs $27-30 \mathrm{~h}$ ), testing the difference from fold-change $=1$. $\boldsymbol{E}$, Firing rate distributions for each replicate at two time points for PTX-treated units from ActD-treated samples: baseline ( -3 to $0 \mathrm{~h}$ ) and post-homeostasis $(27-30 \mathrm{~h})$. $q$ values represent FDR-adjusted Kolmogorov-Smirnov test comparing indicated distributions. $\boldsymbol{F}$, Same as $\boldsymbol{E}$ but for FLA-treated samples. $\mathbf{G}$, The fold-change in firing rate over the course of the assay for ActD experiments. DMSO was added at time 0 . Lighter lines are medians from each of $n=4$ biological replicates (units/replicate: Control, 78-187; ActD: 129-214). Darker lines represent the mean of the median firing rates for replicates from each genotype. $P$ values shown from at test on log(fold-change in firing rate) comparing control and inhibitor-treated cultures. $\boldsymbol{H}$, The fold-change in firing rate over the course of the assay when $A c t D$ was added at $-0.5 \mathrm{~h}$, and then either DMSO or PTX was added at time 0 . Lighter lines are medians from individual replicates ( $n=4$ biological replicates; same data as in $\mathbf{C}$ and $\mathbf{G}$ ). Darker lines represent the mean of the median firing rates for replicates from each genotype. $P$ values shown from a $t$ test on $\log$ (foldchange in firing rate) comparing DMSO- and PTX-treated cultures. $I$, Like $\mathbf{G}$ but for FLA-treated cultures. $n=3$ biological replicates (units/replicate: Control, 60-169; FLA: 29-172). Data provided in Figure 7-1, available at https://doi.org/10.1523/JNEUROSCI.1108-19.2019.F7-1.
}

al., 2014; Mardinly et al., 2016; Hartzell et al., 2018). However, there are many other potentially transcription-independent E/I balance regulation mechanisms. We thus suspect that $\mathrm{E} / \mathrm{I}$ balance could regulate the firing rate homeostasis we observe.

Finally, firing rate homeostasis could be achieved via several additional forms of homeostatic plasticity, including presynaptic plasticity and alterations in Hebbian plasticity. First, the probability of presynaptic vesicle release onto excitatory neurons is altered homeostatically, and this alteration occurs in neurons that undergo firing rate homeostasis (Burrone et al., 2002). Presynaptic homeostasis can occur in a population of synapses onto an individual dendrite, rather than at all synapses onto a neuron, making it a good candidate for ARG-independent homeostasis because dendrite- or synapse-specific homeostasis is less likely to involve the nucleus (Yu and Goda, 2009). In addition, neurons in our experiments may adapt their firing rates by altering their threshold for Hebbian plasticity (Bienenstock et al., 1982; Fernandes and Carvalho, 2016). However, we note that alterations in the threshold for Hebbian plasticity have been suggested, but not conclusively demonstrated, to require Arc (Shepherd and Bear, 2011), which is not required for firing rate homeostasis. Although we do not know what cellular mechanisms underlie firing rate homeostasis in our experiments, our results demonstrate that at least one of them must be able to operate in the absence of transcriptional induction.

\section{Transcription-dependent homeostasis in other contexts}

Although we find that neurons undergo firing rate homeostasis in the absence of activity-regulated transcription in response to persistent PTX stimulation, it is possible that a loss of activityregulated transcription may impair firing rate homeostasis in other contexts. Indeed, different homeostatic mechanisms are engaged in response to different alterations in neuronal activity (Bridi et al., 2018; Kulik et al., 2019). Therefore, some alterations in circuit input might induce transcription-dependent mechanisms of firing rate homeostasis. For example, given that several ARGs have been implicated in regulation of inhibitory synapses onto excitatory neurons (Bloodgood et al., 2013; Spiegel et al., 2014; Hartzell et al., 2018; Gray and Spiegel, 2019), the ARG program may be more effective, and thus important, in regulating firing rate homeostasis in response to perturbations that, unlike PTX, do not themselves block inhibition onto excitatory neurons. Furthermore, neurons may have redundant mechanisms to achieve firing rate homeostasis, and thus require transcription only when other mechanisms are impaired. Consistent with this idea, neurons can achieve the same firing rates and firing patterns with many different ion channel compositions, suggesting there are multiple ways to adjust ion channel use to achieve firing rate homeostasis (Marder and Goaillard, 2006). Therefore, activityregulated-transcription-dependent mechanisms of achieving firing rate homeostasis could be operating in our assay, but neurons may be able to compensate for their loss with transcription-independent mechanisms.

It is also possible that we failed to observe a role for transcription in regulating firing rate homeostasis because firing rate is not the activity parameter that transcription-dependent mechanisms of homeostatic plasticity regulate. For example, criticality is a network-level property of neuronal circuits that describes circuits that have stable activity, i.e., activity that neither dies out nor increases over time (Shew and Plenz, 2013). A recent study demonstrated that in response to visual deprivation, the mouse visual cortex initially enters a subcritical state but returns to criticality over a period of $48 \mathrm{~h}$, which is faster than the cortex undergoes 
firing rate homeostasis in response to the same perturbation ( $\mathrm{Ma}$ et al., 2019). Modeling suggests that homeostasis of criticality could be mediated by transcription-dependent synaptic scaling, raising the possibility that transcription-dependent homeostatic plasticity controls criticality rather than firing rate. In addition to firing rate and criticality, neurons also undergo homeostasis of firing pattern (Marder and Goaillard, 2006), and transcription could also potentially regulate firing pattern homeostasis, for example through regulation of ion channel expression. Our work thus does not rule out a role for activity-regulated transcription in neuronal activity homeostasis, but raises the possibility that to identify its role, it may be important to consider parameters of neuronal activity other than firing rate.

\section{References}

Bateup HS, Denefrio CL, Johnson CA, Saulnier JL, Sabatini BL (2013) Temporal dynamics of a homeostatic pathway controlling neural network activity. Front Mol Neurosci 6:28.

Benevento M, Iacono G, Selten M, Ba W, Oudakker A, Frega M, Keller J, Mancini R, Lewerissa E, Kleefstra T, Stunnenberg HG, Zhou H, van Bokhoven H, Nadif Kasri N (2016) Histone methylation by the Kleefstra syndrome protein EHMT1 mediates homeostatic synaptic scaling. Neuron 91:341-355.

Bienenstock EL, Cooper LN, Munro PW (1982) Theory for the development of neuron selectivity: orientation specificity and binocular interaction in visual cortex. J Neurosci 2:32-48.

Bloodgood BL, Sharma N, Browne HA, Trepman AZ, Greenberg ME (2013) The activity-dependent transcription factor Npas4 regulates domainspecific inhibition. Nature 503:121-125.

Bridi MC, de Pasquale R, Lantz CL, Gu Y, Borrell A, Choi SY, He K, Tran T, Hong SZ, Dykman A, Lee HK, Quinlan EM, Kirkwood A (2018) Two distinct mechanisms for experience-dependent homeostasis in vivo. Nat Neurosci 21: 843-850.

Burrone J, O’Byrne M, Murthy VN (2002) Multiple forms of synaptic plasticity triggered by selective suppression of activity in individual neurons. Nature 420:414-418.

Buzsáki G, Mizuseki K (2014) The log-dynamic brain: how skewed distributions affect network operations. Nat Rev Neurosci 15:264-278.

Chang MC, Park JM, Pelkey KA, Grabenstatter HL, Xu D, Linden DJ, Sutula TP, McBain CJ, Worley PF (2010) Narp regulates homeostatic scaling of excitatory synapses on parvalbumin-expressing interneurons. Nat Neurosci 13:1090-1097.

Chowdhury S, Shepherd JD, Okuno H, Lyford G, Petralia RS, Plath N, Kuhl D, Huganir RL, Worley PF (2006) Arc/Arg3.1 interacts with the endocytic machinery to regulate AMPA receptor trafficking. Neuron 52:445459.

Cho JH, Huang BS, Gray JM (2016) RNA sequencing from neural ensembles activated during fear conditioning in the mouse temporal association cortex. Sci Rep 6:31753.

Diering GH, Nirujogi RS, Roth RH, Worley PF, Pandey A, Huganir RL (2017) Homer la drives homeostatic scaling-down of excitatory synapses during sleep. Science 355:511-515.

Fernandes D, Carvalho AL (2016) Mechanisms of homeostatic plasticity in the excitatory synapse. J Neurochem 139:973-996.

Fleischmann A, Hvalby O, Jensen V, Strekalova T, Zacher C, Layer LE, Kvello A, Reschke M, Spanagel R, Sprengel R, Wagner EF, Gass P (2003) Impaired longterm memory and NR2A-type NMDA receptor-dependent synaptic plasticity in mice lacking c-Fos in the CNS. J Neurosci 23:9116-9122.

Fong MF, Newman JP, Potter SM, Wenner P (2015) Upward synaptic scaling is dependent on neurotransmission rather than spiking. Nat Commun 6:6339.

Goold CP, Nicoll RA (2010) Single-cell optogenetic excitation drives homeostatic synaptic depression. Neuron 68:512-528.

Gray JM, Spiegel I (2019) Cell-type-specific programs for activity-regulated gene expression. Curr Opin Neurobiol 56:33-39.

Grubb MS, Burrone J (2010) Activity-dependent relocation of the axon initial segment fine-tunes neuronal excitability. Nature 465:1070-1074.

Hartzell AL, Martyniuk KM, Brigidi GS, Heinz DA, Djaja NA, Payne A, Bloodgood BL (2018) NPAS4 recruits CCK basket cell synapses and enhances cannabinoid-sensitive inhibition in the mouse hippocampus. eLife 7:e35927.
Held RG, Liu C, Kaeser PS (2016) ELKS controls the pool of readily releasable vesicles at excitatory synapses through its $\mathrm{N}$-terminal coiled-coil domains. eLife 5:e14862.

Hengen KB, Lambo ME, Van Hooser SD, Katz DB, Turrigiano GG (2013) Firing rate homeostasis in visual cortex of freely behaving rodents. Neuron 80:335-342.

Hengen KB, Torrado Pacheco A, McGregor JN, Van Hooser SD, Turrigiano GG (2016) Neuronal firing rate homeostasis is inhibited by sleep and promoted by wake. Cell 165:180-191.

Hu JH, Park JM, Park S, Xiao B, Dehoff MH, Kim S, Hayashi T, Schwarz MK, Huganir RL, Seeburg PH, Linden DJ, Worley PF (2010) Homeostatic scaling requires group I mGluR activation mediated by Homer la. Neuron 68:1128-1142.

Ibata K, Sun Q, Turrigiano GG (2008) Rapid synaptic scaling induced by changes in postsynaptic firing. Neuron 57:819-826.

Johnston GA, Chebib M, Duke RK, Fernandez SP, Hanrahan JR, Hinton T, Mewett KN (2009) Herbal products and GABA receptors In: Encyclopedia of Neuroscience (Squire LR, ed), pp 1095-1101. Oxford UK: Academic.

Kenner L, Hoebertz A, Beil FT, Beil T, Keon N, Karreth F, Eferl R, Scheuch H, Szremska A, Amling M, Schorpp-Kistner M, Angel P, Wagner EF (2004) Mice lacking JunB are osteopenic due to cell-autonomous osteoblast and osteoclast defects. J Cell Biol 164:613-623.

Kuba H, Oichi Y, Ohmori H (2010) Presynaptic activity regulates $\mathrm{Na}+$ channel distribution at the axon initial segment. Nature 465:1075-1078.

Kulik Y, Jones R, Moughamian AJ, Whippen J, Davis GW (2019) Dual separable feedback systems govern firing rate homeostasis. eLife 8:e45717.

Kuzniewska B, Nader K, Dabrowski M, Kaczmarek L, Kalita K (2016) Adult deletion of SRF increases epileptogenesis and decreases activity-induced gene expression. Mol Neurobiol 53:1478-1493.

Lee KY, Chung HJ (2014) NMDA receptors and L-type voltage-gated $\mathrm{Ca}^{2+}$ channels mediate the expression of bidirectional homeostatic intrinsic plasticity in cultured hippocampal neurons. Neuroscience 277:610-623.

Lösing P, Niturad CE, Harrer M, Reckendorf CMZ, Schatz T, Sinske D, Lerche H, Maljevic S, Knöll B (2017) SRF modulates seizure occurrence, activity induced gene transcription and hippocampal circuit reorganization in the mouse pilocarpine epilepsy model. Mol Brain 10:30.

Malik AN, Vierbuchen T, Hemberg M, Rubin AA, Ling E, Couch CH, Stroud H, Spiegel I, Farh KK, Harmin DA, Greenberg ME (2014) Genome-wide identification and characterization of functional neuronal activitydependent enhancers. Nat Neurosci 17:1330-1339.

Mao W, Salzberg AC, Uchigashima M, Hasegawa Y, Hock H, Watanabe M, Akbarian S, Kawasawa YI, Futai K (2018) Activity-induced regulation of synaptic strength through the chromatin reader L3mbtll. Cell Rep 23: 3209-3222.

Marder E, Goaillard JM (2006) Variability, compensation and homeostasis in neuron and network function. Nat Rev Neurosci 7:563-574.

Mardinly AR, Spiegel I, Patrizi A, Centofante E, Bazinet JE, Tzeng CP, Mandel-Brehm C, Harmin DA, Adesnik H, Fagiolini M, Greenberg ME (2016) Sensory experience regulates cortical inhibition by inducing IGF1 in VIP neurons. Nature 531:371-375.

Ma Z, Turrigiano GG, Wessel R, Hengen KB (2019) Critical dynamics are a homeostatic set point of cortical networks in vivo. Neuron. Advance online publication. Retrieved October 7, 2019. doi:10.1016/ j.neuron.2019.08.031.

O'Leary T, Williams AH, Franci A, Marder E (2014) Cell types, network homeostasis, and pathological compensation from a biologically plausible ion channel expression model. Neuron 82:809-821.

Parrish JZ, Kim CC, Tang L, Bergquist S, Wang T, Derisi JL, Jan LY, Jan YN, Davis GW (2014) Krüppel mediates the selective rebalancing of ion channel expression. Neuron 82:537-544.

Porter BE, Lund IV, Varodayan FP, Wallace RW, Blendy JA (2008) The role of transcription factors cyclic-AMP responsive element modulator (CREM) and inducible cyclic-AMP early repressor (ICER) in epileptogenesis. Neuroscience 152:829-836.

Pozzi D, Lignani G, Ferrea E, Contestabile A, Paonessa F, D’Alessandro R, Lippiello P, Boido D, Fassio A, Meldolesi J, Valtorta F, Benfenati F, Baldelli P (2013) REST/NRSF-mediated intrinsic homeostasis protects neuronal networks from hyperexcitability. EMBO J 32:2994-3007.

Ramanan N, Shen Y, Sarsfield S, Lemberger T, Schütz G, Linden DJ, Ginty DD (2005) SRF mediates activity-induced gene expression and synaptic plasticity but not neuronal viability. Nat Neurosci 8:759-767.

Schaukowitch K, Reese AL, Kim SK, Kilaru G, Joo JY, Kavalali ET, Kim TK 
(2017) An intrinsic transcriptional program underlying synaptic scaling during activity suppression. Cell Rep 18:1512-1526.

Seeburg DP, Feliu-Mojer M, Gaiottino J, Pak DT, Sheng M (2008) Critical role of CDK5 and polo-like kinase 2 in homeostatic synaptic plasticity during elevated activity. Neuron 58:571-583.

Shepherd JD, Rumbaugh G, Wu J, Chowdhury S, Plath N, Kuhl D, Huganir RL, Worley PF (2006) Arc/Arg3.1 mediates homeostatic synaptic scaling of AMPA receptors. Neuron 52:475-484.

Shepherd JD, Bear MF (2011) New views of Arc, a master regulator of synaptic plasticity. Nat Neurosci 14:279-284.

Shew WL, Plenz D (2013) The functional benefits of criticality in the cortex. Neuroscientist 19:88-100.

Slomowitz E, Styr B, Vertkin I, Milshtein-parush H (2015) Interplay between population firing stability and single neuron dynamics in hippocampal networks. eLife 4:e04378.

Spiegel I, Mardinly AR, Gabel HW, Bazinet JE, Couch CH, Tzeng CP, Harmin DA, Greenberg ME (2014) Npas4 regulates excitatory-inhibitory balance within neural circuits through cell-type-specific gene programs. Cell 157:1216-1229.

Turrigiano G (2011) Too many cooks? Intrinsic and synaptic homeostatic mechanisms in cortical circuit refinement. Annu Rev Neurosci 34:89-103.
Turrigiano G (2012) Homeostatic synaptic plasticity: local and global mechanisms for stabilizing neuronal function. Cold Spring Harb Perspect Biol 4:a005736.

Turrigiano GG, Leslie KR, Desai NS, Rutherford LC, Nelson SB (1998) Activity-dependent scaling of quantal amplitude in neocortical neurons. Nature 391:892-896.

Tyssowski KM, DeStefino NR, Cho JH, Dunn CJ, Poston RG, Carty CE, Jones RD, Chang SM, Romeo P, Wurzelmann MK, Ward JM, Andermann ML, Saha RN, Dudek SM, Gray JM (2018) Different neuronal activity patterns induce different gene expression programs. Neuron 98:530-546.e11.

Vierbuchen T, Ling E, Cowley CJ, Couch CH, Wang X, Harmin DA, Roberts CWM, Greenberg ME (2017) AP-1 transcription factors and the BAF complex mediate signal-dependent enhancer selection. Mol Cell 68:1067-1082.e12.

Wang KH, Majewska A, Schummers J, Farley B, Hu C, Sur M, Tonegawa S (2006) In vivo two-photon imaging reveals a role of arc in enhancing orientation specificity in visual cortex. Cell 126:389-402.

Yap EL, Greenberg ME (2018) Activity-regulated transcription: bridging the gap between neural activity and behavior. Neuron 100:330-348.

Yu LM, Goda Y (2009) Dendritic signalling and homeostatic adaptation. Curr Opin Neurobiol 19:327-335. 